Historic, archived document

Do not assume content reflects current scientific knowledge, policies, or practices. 


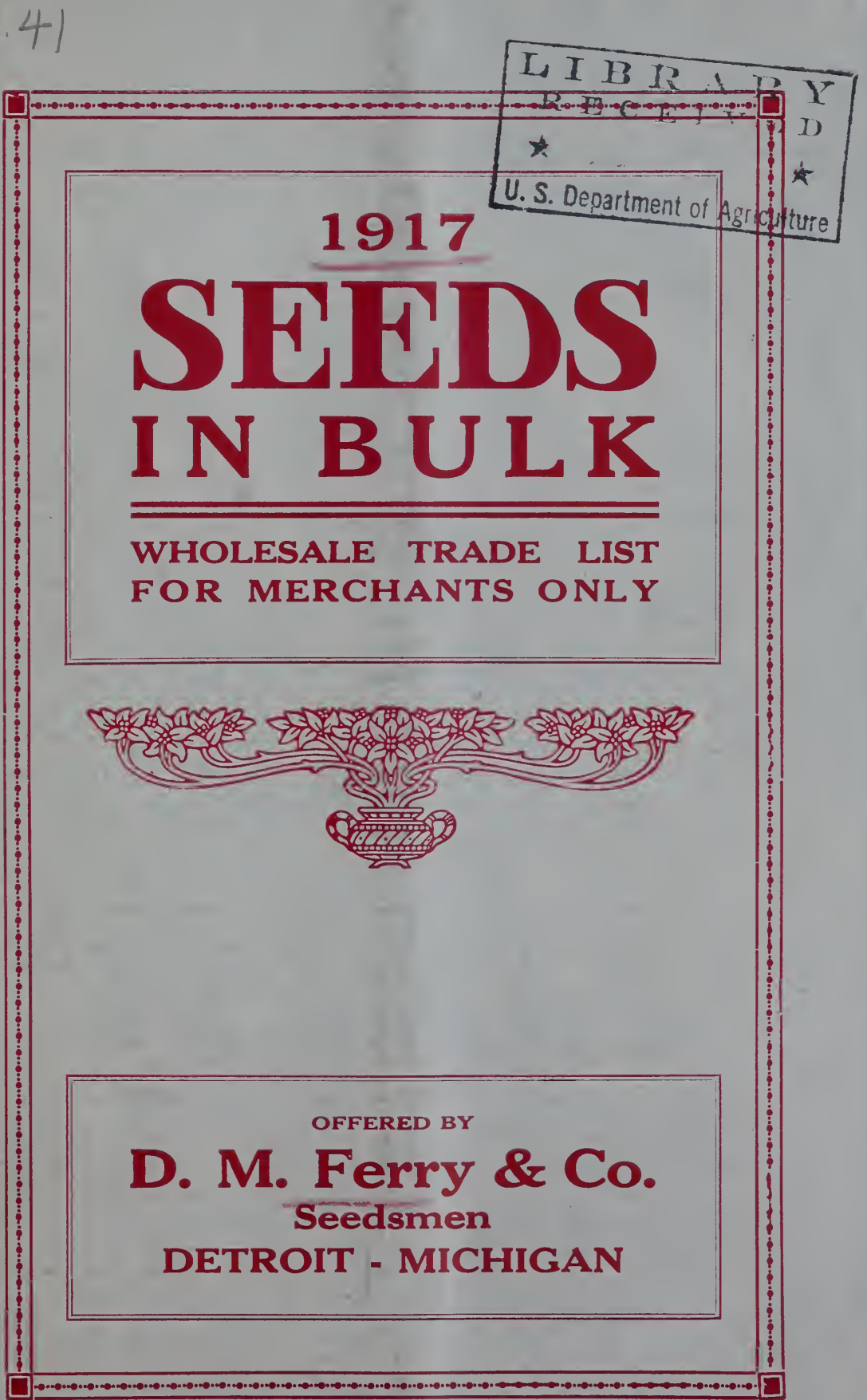




\section{SPECIAL NOTICE}

TT T HANDLE absolutely nothing that we do not believe to be the best obtainable. Our unequaled advantages for the production of the best seeds enable us to offer them to our customers at the lowest prices for which well grown and selected seeds true to name

can be furnished.

Cheap goods in our line should be resolutely shunned by all dealers who want to supply seeds that will satisfy and make money for the planters and gardeners who trust them with their orders. Our seeds have behind them a history of constant success during the past sixty-one years and we continue to affirm-

The best is always the cheapest;

Reliable seeds cannot be afforded at half price.

These prices are made in anticipation of probable results of crops, therefore, we are not bound by them for any definite time or quantity and they are subject to change without notice.

The prices are for delivery f. o. b. cars, Detroit, Mich., and do not include the cost of containers. When seamless cotton grain bags, boxes, or barrels are necessary for safe transportation, such packages are charged at value and are not returnable.

Not less than $25 \mathrm{lbs}$. of Grass, Clover, Miscellaneous Farm Seeds or Bird Seeds at $100 \mathrm{lb}$. rate. On other seeds pound prices apply to quantities of $1 / 4 \mathrm{lb}$. or more, hundred pound prices to $10 \mathrm{lbs}$. or more.

The varieties in each class (for example, Wax Podded Beans) are usually arranged in their order of earliness.

TERMS NET CASH, June 1st, 1917, without regard to date of purchase, or a discount of one per cent. per month for the unexpired time when paid before maturity. Field Peas, all Grass and Clcver Seeds, Miscellaneous Farm Seeds and Bird Seeds, net cash 10 days, without any discount whatever. Cash or satisfactory references should accompany orders from unknown correspondents.

We give no warranty, express or implied, as to description, purity, productiveness, or any other matter of any seeds we send out, and we will not be in any way responsible for the crop. If the purchaser does not accept the goods on these terms they are at once to be returned.

\section{M. FERRY \& CO.}

DETROIT, MICH., January 25, 1917.

In telegraphing, please use our CODE on page 49. Our telegraph cipher words should be used in Telegrams only-not in Orders by Mail. 


\section{EQUIVALENT BUSHEL QUOTATIONS}

For the convenience of those of our customers who have not yet become accustomed to using the Cental System in connection with prices of Beans, Corn and Peas, we subjoin a table which will enable one at a glance to find the equivalent price per bushel of any given price per hundred pounds.

Weights of measured bushels always vary somewhat. Corn ranges from 40 to $50 \mathrm{lbs}$. per bu.; we have made our table for the average, i. e., $45 \mathrm{lbs}$. Wrinkled Peas and Field Corn weigh 56 lbs. per bu., Beans and Smooth Peas, 60 lbs. per bu.

\begin{tabular}{|c|c|c|c|c|c|c|c|c|c|c|c|c|c|c|c|}
\hline $\begin{array}{l}\text { Per } \\
100 \\
\text { Ibs. }\end{array}$ & $\begin{array}{l}\text { Per } \\
45 \mathrm{Ib} \text {. } \\
\text { bush. }\end{array}$ & \begin{tabular}{|c|c|} 
& Per \\
56 & Ib. \\
bush.
\end{tabular} & $\begin{array}{c}\text { Per } \\
60 \text { Ib. } \\
\text { bush. }\end{array}$ & $\begin{array}{l}\text { Per } \\
100 \\
\text { lbs. }\end{array}$ & $\begin{array}{c}\text { Per } \\
45 \mathrm{Ib} \text {. } \\
\text { bush. }\end{array}$ & $\begin{array}{c}\text { Per } \\
56 \text { Ib. } \\
\text { bush. }\end{array}$ & $\begin{array}{c}\text { Per } \\
60 \text { lb. } \\
\text { bush. }\end{array}$ & $\begin{array}{l}\text { Per } \\
100 \\
\text { Ibs. }\end{array}$ & $\begin{array}{c}\mathrm{Per} \\
45 \mathrm{Ib} \text {. } \\
\text { bush. }\end{array}$ & $\begin{array}{c}\text { Per } \\
56 \text { Ib. } \\
\text { bush. }\end{array}$ & $\begin{array}{c}\text { Per } \\
60 \mathrm{Ib} . \\
\text { bush. }\end{array}$ & $\begin{array}{l}\text { Per } \\
100 \\
\text { lbs. }\end{array}$ & $\begin{array}{c}\text { Per } \\
45 \mathrm{Ib} . \\
\text { bush. }\end{array}$ & $\begin{array}{c}\text { Per } \\
56 \text { Ib. } \\
\text { bush. }\end{array}$ & \begin{tabular}{|l} 
Per \\
60 lb. \\
bush.
\end{tabular} \\
\hline .00 & .45 & $\$ .56$ & s .60 & $\$ 15.00$ & $\$ 6.75$ & $\$ 8.40$ & $\$ 9.00$ & 29.00 & $\$ 13.0$ & 16.24 & $\$ 17.40$ & 43.00 & $\$ 19.3$ & 24.08 & 5.80 \\
\hline & & & & 15.25 & 6.86 & 8.54 & 9.15 & 29.25 & $\begin{array}{l}13.15 \\
\end{array}$ & & 17.55 & $\begin{array}{l}73.250 \\
43.25\end{array}$ & 19.46 & 24.22 & \\
\hline 1. & .68 & .84 & .90 & 15.50 & 6.98 & 8.68 & 9.30 & 29.50 & 13.28 & 16.52 & 17.70 & 43.50 & 19.58 & .36 & 6.10 \\
\hline 1. & .79 & .98 & 1.05 & 15.75 & 7.09 & & 9.45 & 29.75 & $13.3 y$ & 66 & 17.85 & .75 & 69 & .50 & 6.25 \\
\hline & .90 & 1.12 & 1.20 & 16.00 & 7.20 & 8.96 & 9.60 & 30.00 & 13.50 & 16.80 & 18.00 & 4.00 & & 64 & 26.40 \\
\hline 0 & 1.01 & 1.26 & 1.35 & 16.25 & 7.31 & 9.10 & 9.75 & 30.25 & 13.61 & 16.94 & 18.15 & 44.25 & 19.91 & 24.78 & 26.55 \\
\hline & 1.13 & 1.40 & 1.50 & 16.50 & 7.43 & 9.24 & 9.90 & 30.50 & 13.73 & & 18.30 & & & & 67 \\
\hline 2. & 1.24 & 1.54 & 1.65 & 16.75 & 7.54 & 9.38 & 10.05 & 30.75 & 13.84 & 17.22 & 18.45 & 75 & 20.14 & 5.06 & 26.85 \\
\hline 3. & 1.35 & 1.68 & 1.80 & 17 & 7.65 & 9.52 & 10.20 & 0 & 13.95 & 17.36 & 18.60 & 00 & & 20 & 00 \\
\hline & 1.46 & 1.82 & 1.95 & 17 & 7.76 & 9.66 & 10.35 & 25 & 14.06 & 17.50 & 18.75 & & 20.36 & & 27.15 \\
\hline 3. & 1.58 & 1.96 & 2.10 & & & 9.80 & 10.50 & 31.50 & & & 18.90 & & & 5.48 & 27. \\
\hline 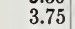 & $\begin{array}{l}1.69 \\
\end{array}$ & 2.10 & 2.25 & 17.7 & 7.99 & 9.94 & 10.65 & 31.75 & 14.29 & 17.78 & 19.05 & & 20.59 & 5.62 & 27 \\
\hline & 1.80 & 2.24 & 2.40 & 18.00 & 8.10 & 10.08 & & & 14 & & & & & .76 & \\
\hline & 1.91 & 2.38 & $? .55$ & & 8.21 & 10.22 & 10.95 & & & & & & & & 75 \\
\hline & 2.03 & 2.52 & 2.70 & 18 & 8.33 & $\begin{array}{l}10.36 \\
\text { S }\end{array}$ & 11.10 & & & & 19.50 & & & .04 & 27.90 \\
\hline & 2.14 & 2.66 & 2.85 & 18. & 8.44 & 10.50 & & & & & & & & 18 & 28 \\
\hline & & 2.80 & 3.00 & 19 & 8.55 & .64 & & & & & & & & & \\
\hline & 2.36 & 2.94 & 3.15 & & 8.66 & 10.78 & & & & & & & & & \\
\hline & 2.48 & 3.08 & $\begin{array}{l}3.10 \\
3.30\end{array}$ & & 8.78 & $\begin{array}{l}10.92 \\
\text {. }\end{array}$ & 11.70 & & & & & & & & 50 \\
\hline & . & & 3.45 & & 8.89 & & & & & & & & & & \\
\hline & 2.70 & 36 & $\begin{array}{l}3.6 \\
3.6\end{array}$ & & 9.00 & 20 & & & & & & & & & \\
\hline & & & 3. & & 9.11 & 34 & & & & & & & & & 95 \\
\hline & 93 & & 3.90 & & 9.23 & 8 & 12.30 & & & & & & & & 0 \\
\hline & 3 & & & & 9.34 & & & & & & & & & & \\
\hline & 3. & ${ }_{3}$ & 4.2 & & 9.45 & .76 & & & & & & & & & \\
\hline & 6 & 4.06 & 4 & & 9.56 & 90 & 12.75 & & & 19 & & & & & 29 . \\
\hline & & & & & & 04 & & & & & & & & & \\
\hline & & & & & & & & & & & & & & & \\
\hline & 3.60 & & 4.8 & & 9.90 & 32 & & & & & & & & & \\
\hline & 1 & & & & 10 & 46 & & & & & & & & 14 & 30 . \\
\hline & & & & & & & & & & & & & & & \\
\hline & & & & & & & & & & & & & & & \\
\hline & 4. & & 5. & & & & & & & & & & & & \\
\hline & & & & & & & & & 1 & & & & & & \\
\hline & & & & & & & & & & & & & & & \\
\hline & & & & & & & & & & & & & & & \\
\hline & & & & & & & & & & & & & & & \\
\hline & & & & & & & & & & & & & & & \\
\hline & & & & & & & & & & & & & & & \\
\hline & & & & & & & & & & & & & & & \\
\hline & & & & & & & & & & & & & & & \\
\hline & & & & & & 4 & & & 17 & & & & & & \\
\hline & & & & & & & & & & & & & & & \\
\hline & & & & & & & & & & & & & & & \\
\hline & & & & & & & & & & & & & & & \\
\hline & & & & & & & & & & & & & & & \\
\hline & & & & & & & & & & & & & & & \\
\hline & & & & & & & & & & & & & & & \\
\hline & & & & & & & & & & & & & & & \\
\hline & & & & & & & & & & & & & & & \\
\hline & & & & & & & & & & & & & & & \\
\hline & & & & & 12. & & & & & & & & & & \\
\hline & & & & & & & & 42 & 18.90 & 23 & & & 20 & 31.36 & \\
\hline & & & & & & & & & 19 & & & & & & \\
\hline & & 8.1 & 8. & 28 & 12.8 & 15. & & 42. & 19. & 23 & & & & 31.64 & 33 . \\
\hline & & & & & 12.9 & 10. & & 72. & 19. & 23. & 25 & 56.75 & 25 . & 31.7 & 21 \\
\hline
\end{tabular}




\section{STANDARD SEEDS IN BULK}

ARTICHOKE

Tel. Cipher PerLb.

Green Globe

\section{ASPARAGUS}

Columbian Mammoth White ...................Adair.....\$0 35

Conover's Colossal. ..........................Aitkin..... 25

Palmetto.......................................... 25

ASPARAGUS ROOTS

Per 1000

Columbian Mammoth White.....

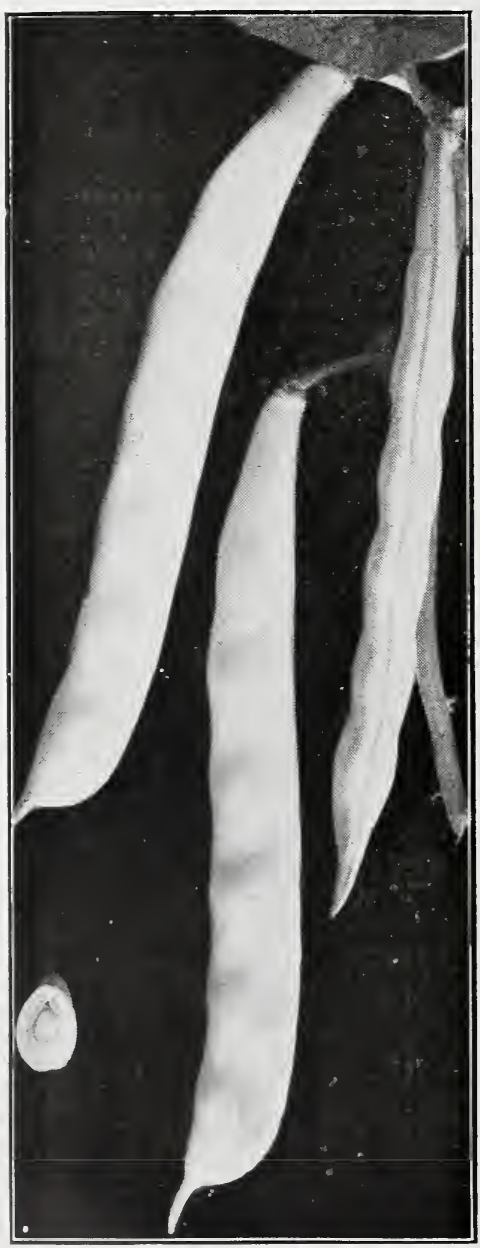

Michigan White WaX
Conover's Colossal..... Alamance.. 450

Palmetto.............Albemarle . 450

\section{BEANS-Dwarf or Bush}

Not less than $10 \mathrm{lbs}$. at $100 \mathrm{lb}$. rate.

WAX PODDED SORTS

Challenge Dwarf

Black Wax. The

earliest wax pod-

ded bean. We con-

Per

sider it the best first Cipher Per Lb. 100 Lbs.

early wax sort.... Albany.\$0 $30 \quad \$ 22 \quad 50$

D. M. Ferry \& Co's

Golden Wax....Alcorn.... $30 \quad 2250$

Michigan White

Wax. (See cut) Alger.... $30 \quad 2500$

Grenell Rust Proof

Wax..........Allegany.. $30 \quad 2250$

Refugee Wax.......Amador.. sold out

Keeniey's Rustless

Golden Wax. .. Alpena... $30 \quad 2250$

D. M. Ferry \& Co's

Detroit Wax.....Amherst.. 252000

Prolific German

Wax, B lack

Seeded........Anderson $30 \quad 2250$

Pencil Pod Wax

(very short crop).. Andrews. crop failed

Currie's Rust Proof

Wax ..........Anoka.... $30 \quad 2500$

Dwarf Unrivaled

Wax.........Anson.... 252000

Wardwell's Kidney

Wax.........Antelope. $30 \quad 2500$

Round Pod Kidney

Wax..........Apache... sold out

Davis Wax. ...Aransas.. $30 \quad 2250$

Scarlet Flageolet

Wax .........Archer... $30 \quad 2500$

Violet Flageolet Wax

or PerfectionWax Arenac... $30 \quad 2500$

Hodson Wax.......Ashe.... sold out

Crystal White Wax.Ashley.... $30 \quad 2500$ 


\section{BEANS-Dwarf or Bush-Continued}

GREEN PODDED SORTS

Tel. Cipher Per Lb. 100 Lbs.

Extra Early Refugee......................Atascosa $\$ 020 \$ 1500$

Round Yellow Six Weeks, Improved Round Pod.....Athens... $20 \quad 200$

Early Yellow Kidney Six Weeks ..................Atoka.... 201400

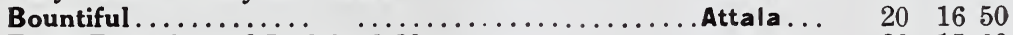

Extra Early Round Pod Red Valentine...........Auglaize. 201500

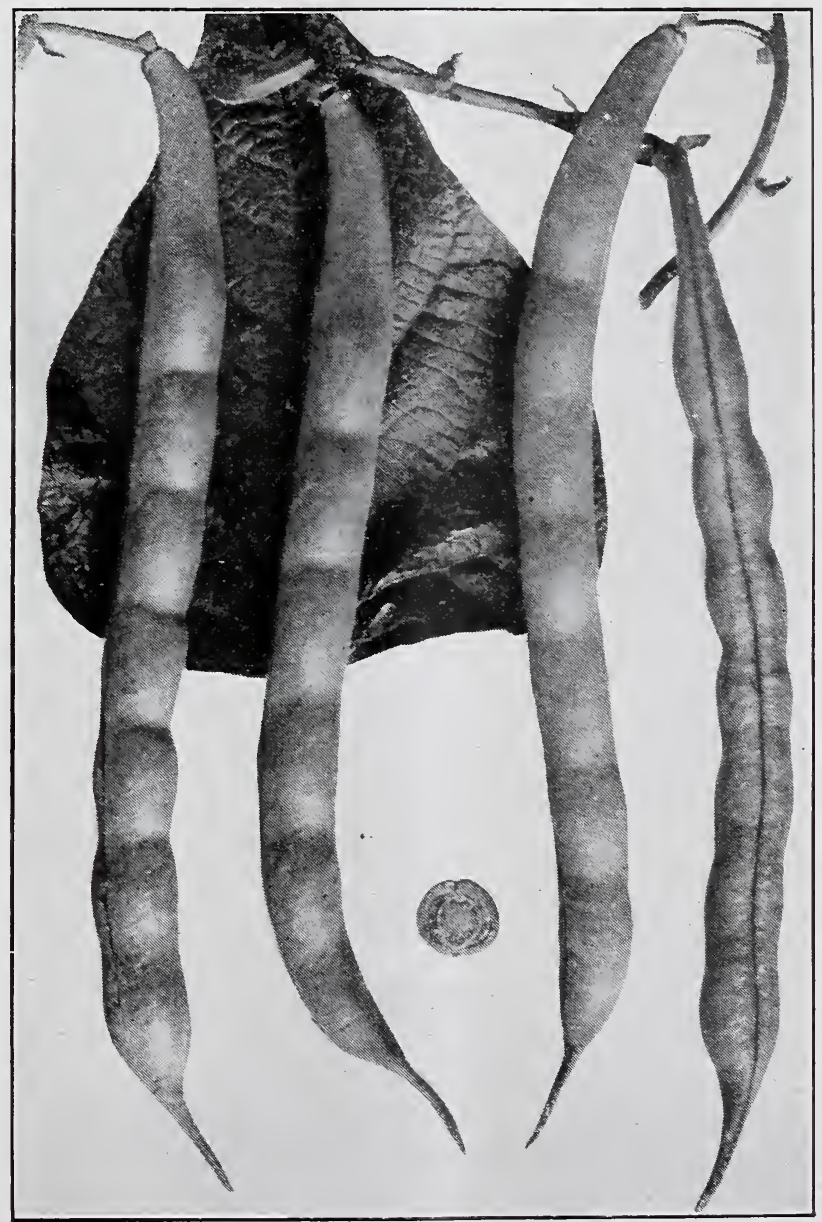

Giant Stringle8s Green Pod 


$$
1
$$




\section{BEANS-Dwarf or Bush-Continued}

Not less than $10 \mathrm{lbs}$. at $100 \mathrm{lb}$. rate.

GREEN PODDED SORTS

Tel.Cipher Per Lb. 100 Lbs.

Black Valentine Autauga..\$0 $20 \$ 1600$

Early Mohawk.......................Audrain...

$20 \quad 1400$

Stringless Green Pod

Bailey....

252000

Giant Stringless Green Pod (See cut,page 3) .......Baldwin.. 252000

Tennessee Green Pod. Pods very long, flat, irregular in shape, bright, rather dark green and of fine quality .. Ba mberg.

Dwarf Horticultural.

Banks...

201600 sold out

Refugee or Thousand to One. Remarkably productive. Stands handling well and is a very popular

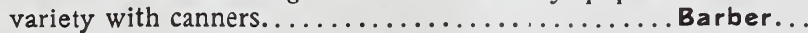

201600

Early Marrow Pea or Dwarf White Navy .................. crop failed

Large White Marrow or Mountain ...................... 151300

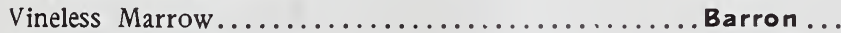
151300

Royal Dwarf Kidney

Bastrop..

crop failed

\section{DWARF LIMAS}

Bush Lima or Dwarf Sieva (Henderson's) ............ Bath ....

Burpee's Improved Bush Lima ............... Bayfield..

Dwarf Large White Lima (Burpee's).............. Baylor ...

Fordhook Bush Lima.

Beadle...

\section{Pole or Running}

White Creaseback.......................... Becker...

Kentucky Wonder. (Old Homestead) Very productive and of the best quality for snaps. (See cut $). . . \ldots \ldots$..... Bedford..

St. Louis Perfection White. Early, very productive, white seeded; unsurpassed in its class............. Beckham.

Red Speckled Cut Short or Corn Hill.............Belknap..

Scotia or Striped Creaseback. Especially suitable for

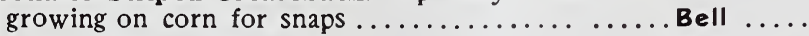

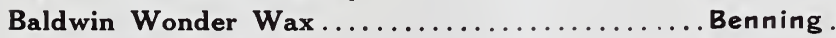

Dutch Case Knife

Belmont.

Kentucky Wonder Wax. Similar to Kentucky Wonder, but pods are attractive light yellow................ Beltrami.

Early Golden Cluster Wax.................Benson ..

London Horticultural or Speckled Cranberry ..........Benzie...

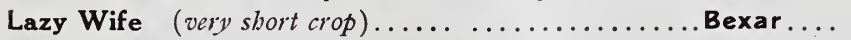

201400 sold out

Seibert's Early Lima.....................Bighorn..

Small White Lima, Carolina or Sieva ............. Billings ..

Ideal Pole Lima ........................ Bladen...

King of the Garden Lima.

Blair.....

Carpinteria Pole Lima. Very large beans $w$. greenish

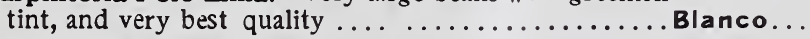




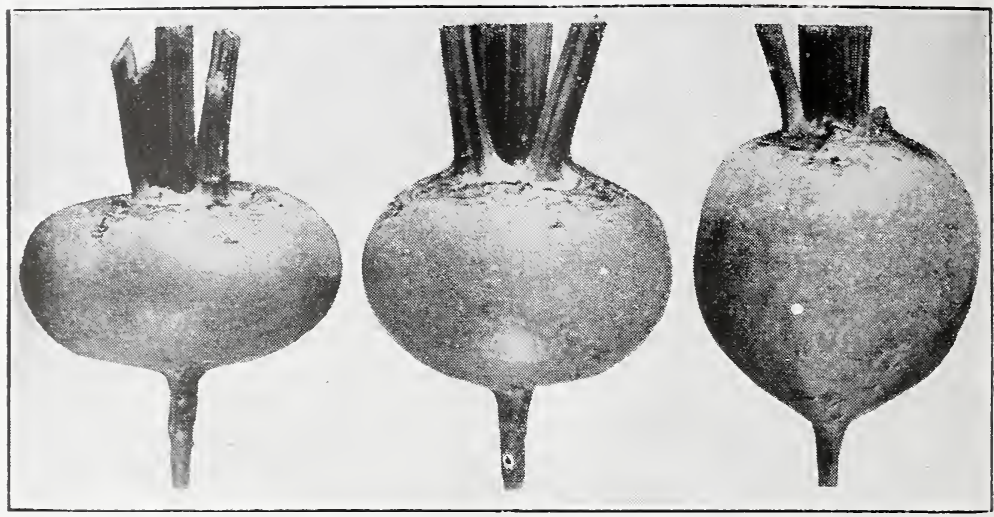

Extra Early Egyptian, Crosby's Egyptian and Detroit Dark Red

\section{BEET}

Tel. Cipher Per Lb.

Extra Early Egyptian Blood Turnip. (See cut)...........Boise ..... \$0 60

Crosby's Egyptian. (See cut) .............................. -190

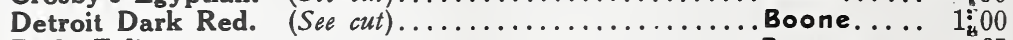

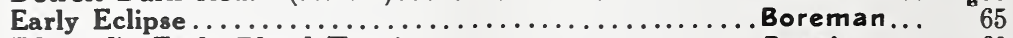

Edmand's Early Blood Turnip ......................... 60

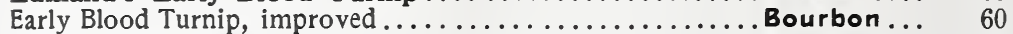

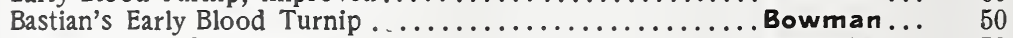

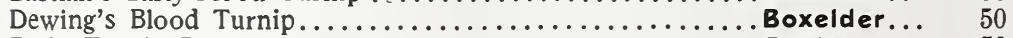

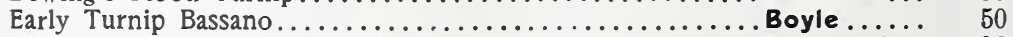

D. M. Ferry \& Co's Half Long Blood............................... 90

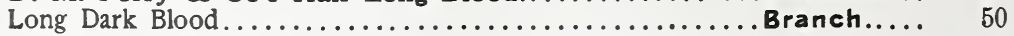

Swiss Chard. Sometimes called Sea Kale Beet or Spinach Beet. . Brazoria... 75

SUGAR BEET Tel. Cipher Per Lb. $100 \mathrm{Lbs}$

Jaensch's Victrix, For Sugar Making..........Bremer...\$0 40 \$35 00

Vilmorin's Improved, " " $"$ " .............. Brewster... $40 \quad 3500$

Klein Wanzleben, " " $"$ "............. Bristol..... $40 \quad 3500$

Giant Feeding Sugar Beet or Half Sugar Mangel

For Stock Feeding............................. $35 \quad 3000$

\section{MANGEL WURZEL}

Long Red................................. $35 \quad 3000$

D. M. Ferry \& Co's Imp'd Mammoth Long Red. Buncombe. $\quad 35 \quad 3000$

Yellow Leviathan ............................. $35 \quad 3000$

Golden Tankard............................ $40 \quad 3500$

\section{BROCCOLI}

\section{BRUSSELS SPROUTS}

Improved Half Dwarf........................ Cabell . 


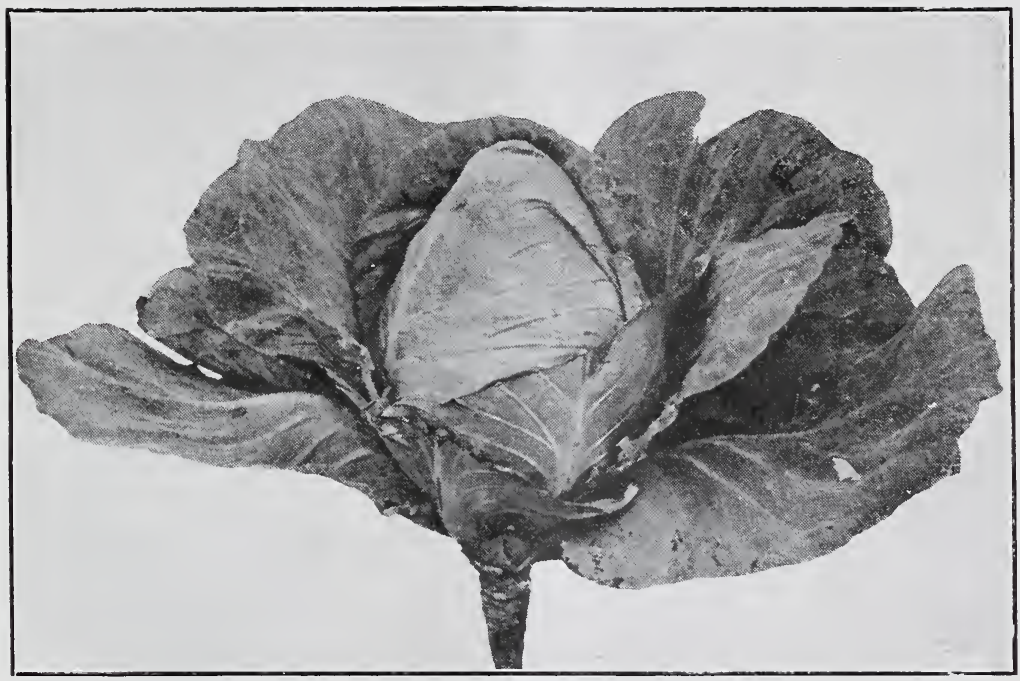

EARLY JERSEY WAKEFIELD

\section{CABBAGE}

For many years Cabbage Seed has been a leading specialty with us. Our cabbage seeds are all grown from approved stock seeds which are largely of our own development on our Oakview Stock Seed Farm and are all grown under our personal inspection and supervision. There is none more reliable, none that can be more implicitly depended upon to give planters uniformly satisfactory results.

\section{FIRST EARLY SORTS}

Tel. Cipher Per Lb.

Early Jersey Wakefield. (See cut) The earliest and surest heading of first early cabbages........................... \$1 75

Copenhagen Market. The earliest large round-headed cabbage. Our strain produces the characteristic large, round heads that for their solidity and excellent quality make this

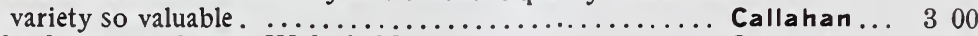

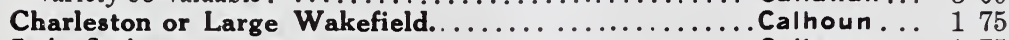

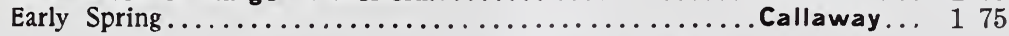

\section{SECOND EARLY AND INTERMEDIATE SORTS}

Early Dwarf Flat Dutch............................. 150

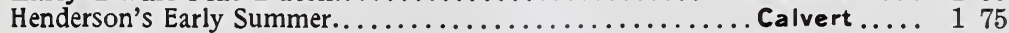

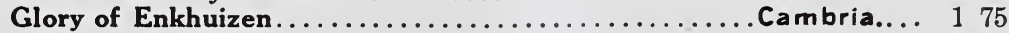

All Head Early................................. 250

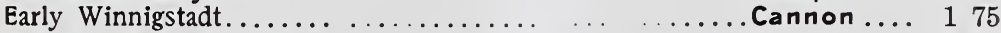

Fottler's Early Drumhead or Brunswick Short Stem .....Carroll..... 125

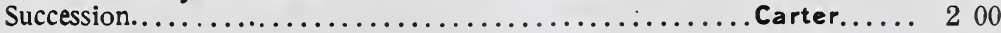

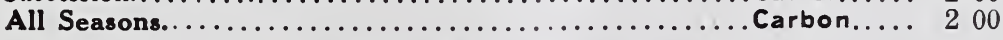

\section{LATE OR AUTUMN AND WINTER SORTS}

The Warren or Improved Stone Mason................... 175

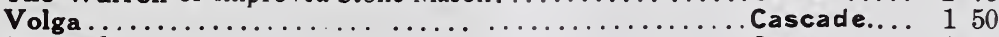

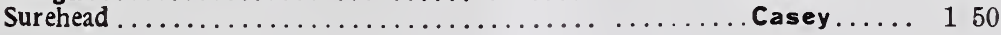




\section{CABBAGE-Continued}

LATE OR AUTUMN AND WINTER SORTS Tel. Cipher Per Lb.

D. M. Ferry \& Co's Premium Late Flat Dutch. ........Catoosa ...... \$1 50

D. M. Ferry \& Co's Premium Late Drumhead .........Chambers.... 150 Hollander or Danish Ball Head. Our own strain and

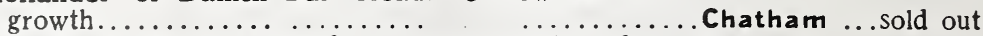
Hollander or Danish Ball Head. Choice Danish seed..... Chaves....... 175

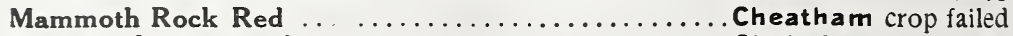
Large Red Drumhead ...................... Chehalis.... 250 Savoy, Early Dwarf Ulm .............................. 150 Savoy, Improved American......................... 150

CHINESE IMPROVED or PE-TSAI. Also called Celery Cabbage. Grows like Cos Lettuce........................... 250

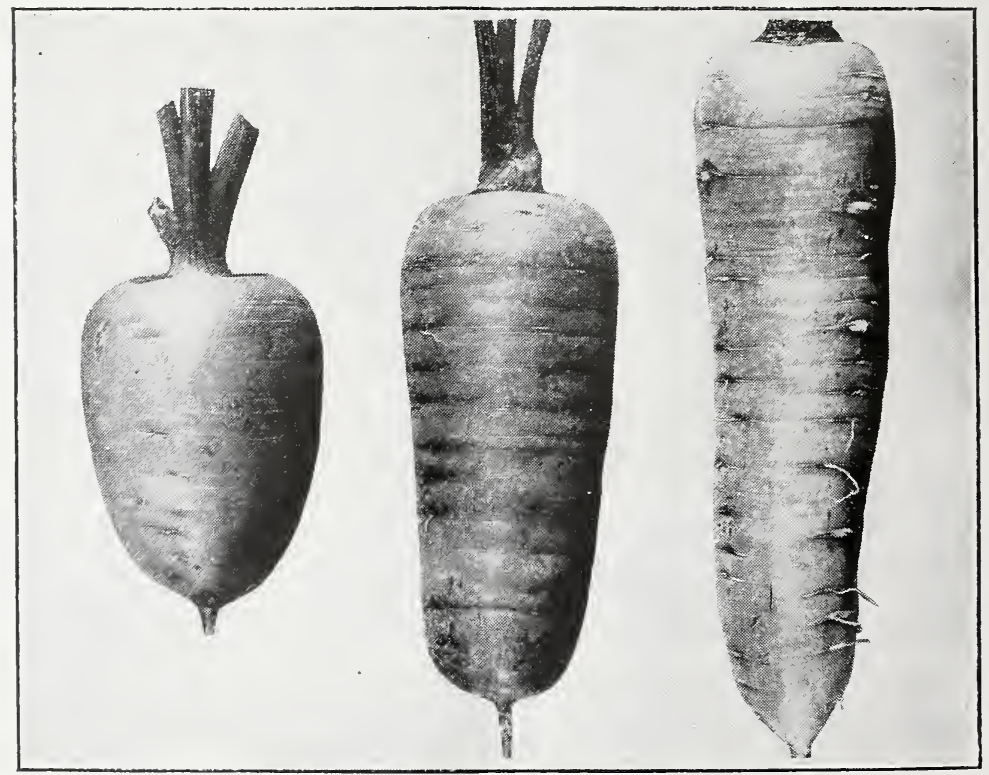

Guerande, Chantenay and Danvers

CARROT

Tel. Cipher Per Lb.

Earliest Short Horn, for forcing

Chester.... \$150

Early Scarlet Horn......................................... 125

Early Half Long Scarlet Carentan, coreless............................ 150

Half Long Scarlet Nantes, Stump-Rooted.................. Choctaw ... 150

Chantenay. (See cut)........................................ 125

Guerande or Ox Heart. (See cut).... ..........Clare..... 100

Danvers. Our strain is unsurpassed. (See cut).......... Clark...... 125

Improved Long Orange ...................... Clatsop .... 100

D. M. Ferry \& Co's Improved Short White. ............ Clayton .... 50 


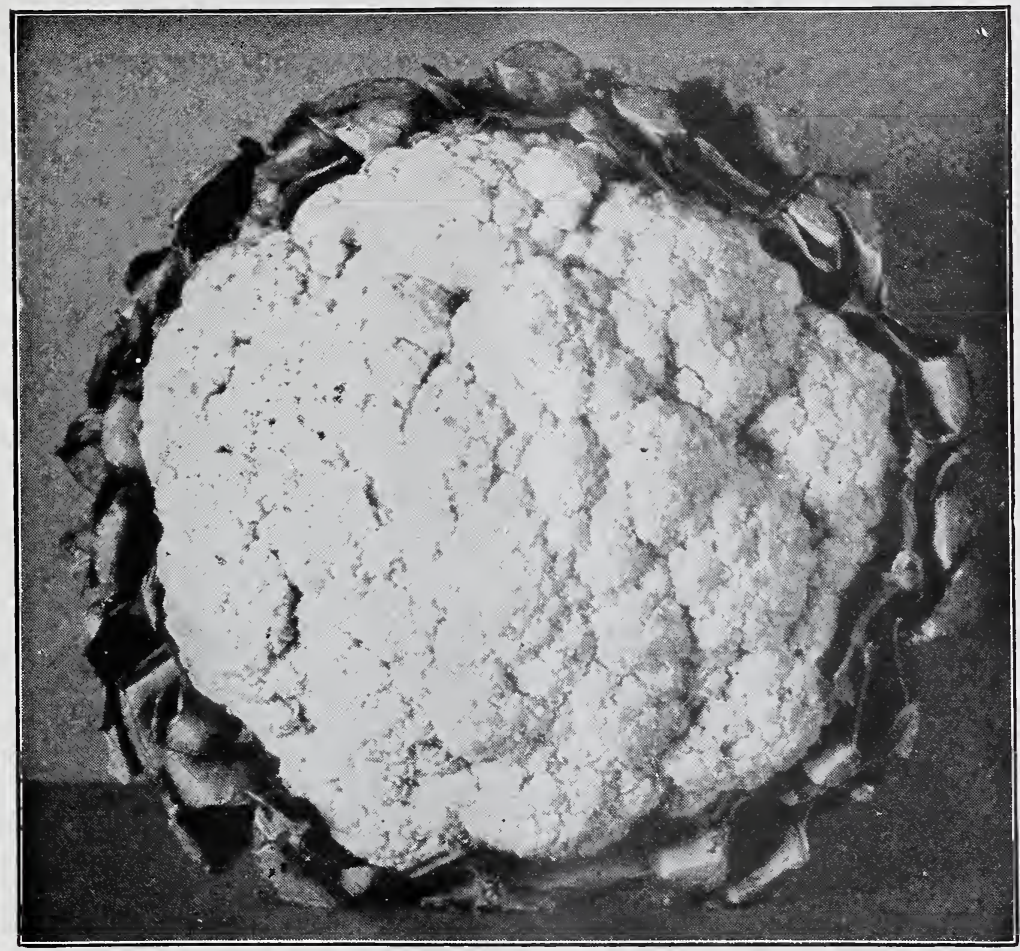

EARLY SNOWBALL

\section{CAULIFLOWER}

Our stocks of Cauliflower are all of the finest quality and are produced by the most experienced growers in Europe, in such localities as are best suited to their proper develop ment and the greatest care is taken to save seed from perfectly developed plants only.

Early Snowball. This is unquestionably the earliest Tel. Cipher Per Oz. Per Lb. maturing strain of the true short leaved Snowball type. The heads are of medium to large size, round and solid, with compact, very white curd of the very best quality. (See cut)........................Clermont.. $\$ 150 \quad \$ 2000$

Extra Early Dwarf Erfurt. A remarkably sure heading early cauliflower. It is nearly as early as Snowball. The heads when ready for market are of medium to large size, curd white, exceptionally deep and of finest quality..................... Clinton... 125

Early Favorite. A large, exceptionally sure heading and easily grown, second early variety...................... $50 \quad 500$ Large Algiers........................................ $40 \quad 450$ Veitch's Autumn Giant .......................Coffee.... $30 \quad 350$ 
D. M. FERRY \& CO., DETROIT, MICH.

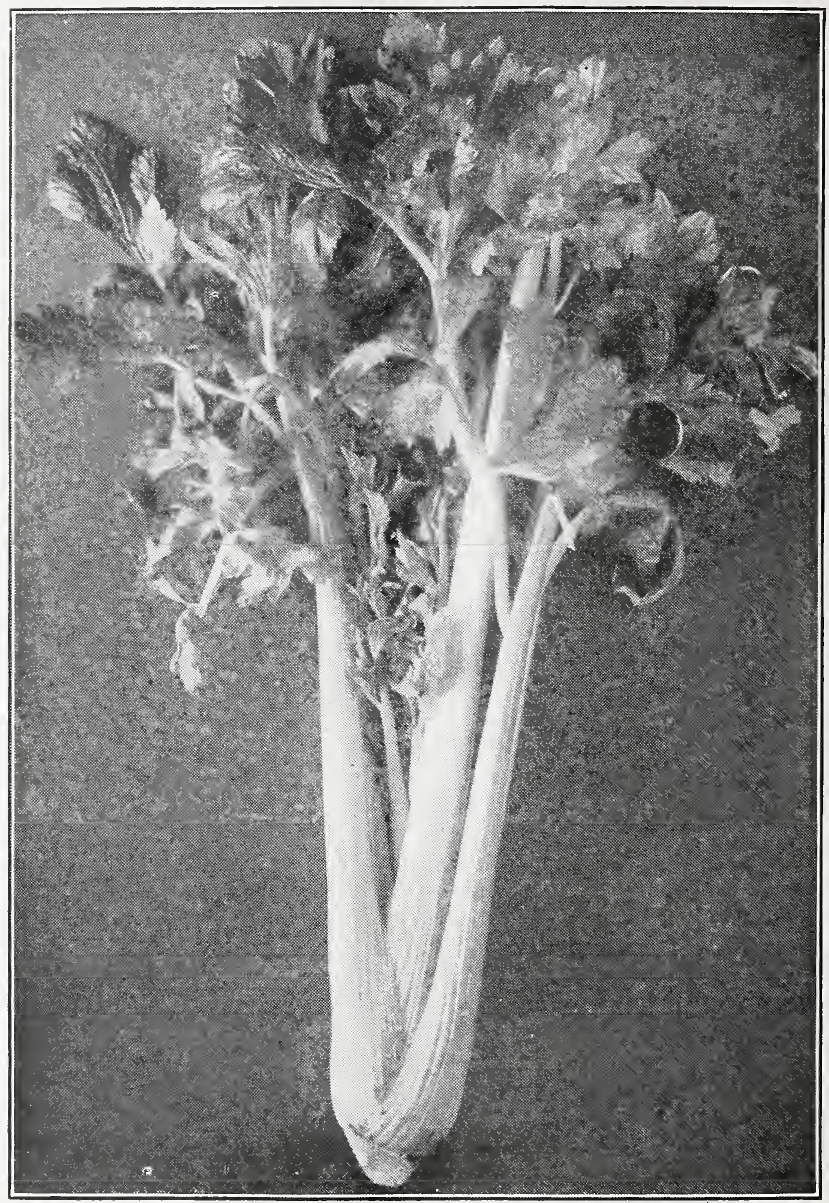

Golden Yellow Self Blanching

CELERY

Tel. Cipher

Per Lb.

Golden Yellow Self Blanching. (See cut) Oz. $\$ 100$. Coke.... $\$ 1200$

White Plume Finest strain . (See aut) Oz.

Columbia. One of the earliest maturing varieties.

Introduced by us in 1906. In rich nutty flavor our

strain is unsurpassed...................... Colleton .......

Dwarf White Solid or Large Ribbed Kalamazoo....... Collin..........

Giant Pascal .................................

Winter Reliance. A very desirable late keeping variety Conejos.......

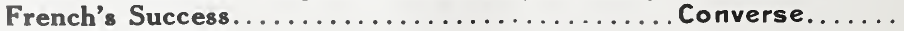

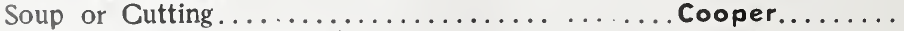

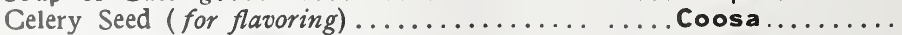

175

500

150

125

250

250

CELERIAC, Large Smooth Prague............Cortland...... 200 


\section{CHERVIL}

Tel. Cipher Per Lb.

Curled CHICORY Oz. $\$ 0.10$. Costilla.. \$075

Large Rooted or Coffee.

CHIVES

..Coweta. . 100

Allium Schœnoprasum .

COLLARDS

Per Oz.

Per Lb.

Georgia, Southern or Creole..

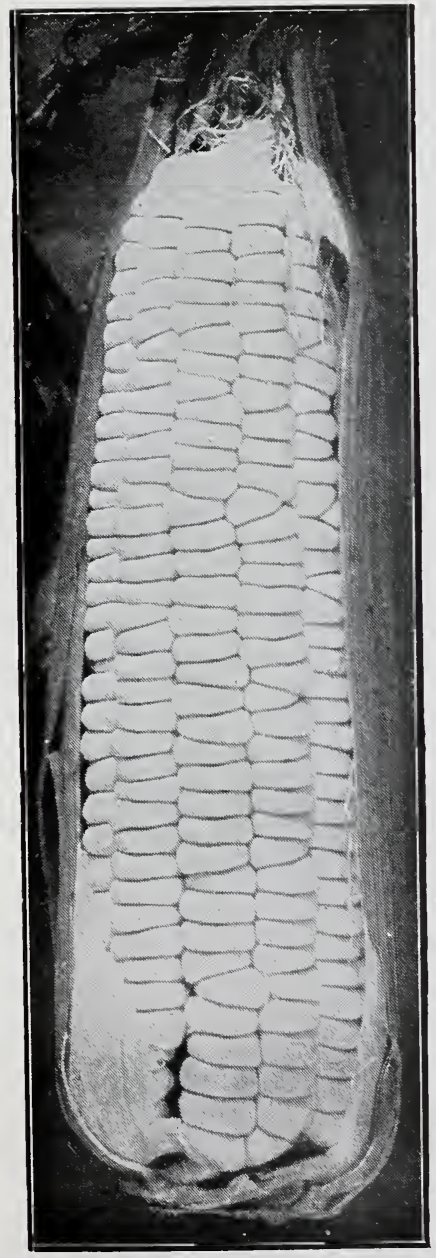

MAMMOTH WhITE CORY

\section{CORN}

(SHELLED)

CAUTION-Sweet Corn in bulk, even after being thoroughly cured, will often gather moisture and spoil, particularly if it has been exposed to a low temperature. To prevent this it should be taken out of the bags as soon as received and spread in a dry place.

Not less than 10 lbs. at $100 \mathrm{lb}$. rate.

Sweet Corn crops this year were either very short or total failures. Our supplies are meager and consequently the prices we quote are not only high but also are for moderate quantities only.

\section{SWEET OR TABLE VARIETIES Per}

Tel. Cipher Per Lb. 100 Lbs.

Mammoth White Cory..Crawford. $\$ 020 \$ 1500$

Crosby's Early .........Cross.... 302500

Golden Bantam. Not our own growth. Purchased

after harvest .........Culpeper. 302500

Early Minnesota........ Curry.....

Kendel's Early Giant.......Cuyahoga

Early Sweet or Sugar...... Dakota...

Black Mexican.......... Darke....

Ferry's Early Evergreen. Davidson.

Avon Evergreen........ Dauphin .

Country Gentleman.....Davis.....

Stowell's Evergreen......Dawes...

Mammoth .............. Day.....

FIELD VARIETIES

Extra Early Adams......... Decatur.. $10 \quad 600$

Early Adams or Burlington Dekalb... $10 \quad 600$

Early Red Blazed........... Denton... $05 \quad 350$

Early Golden Dent or Pride of the North...........Denver.... $05 \quad 350$

\section{POP CORN}

Black Beauty (on the cob) Desha.... (shelled)............. 10

White Rice (on the cob). Deuei....

" " (shelled) ............ 10

\section{CORN SALAD or Fetticus}

Large Seeded, Large Leaved, extra cleaned............Dewitt........ \$0 35 
D. M. FERRY \& CO., DETROIT, MICH.

\section{CRESS}

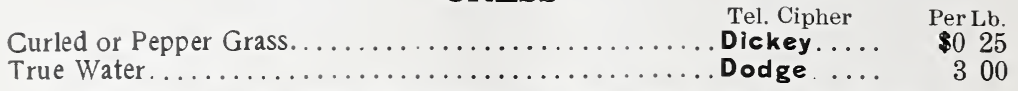

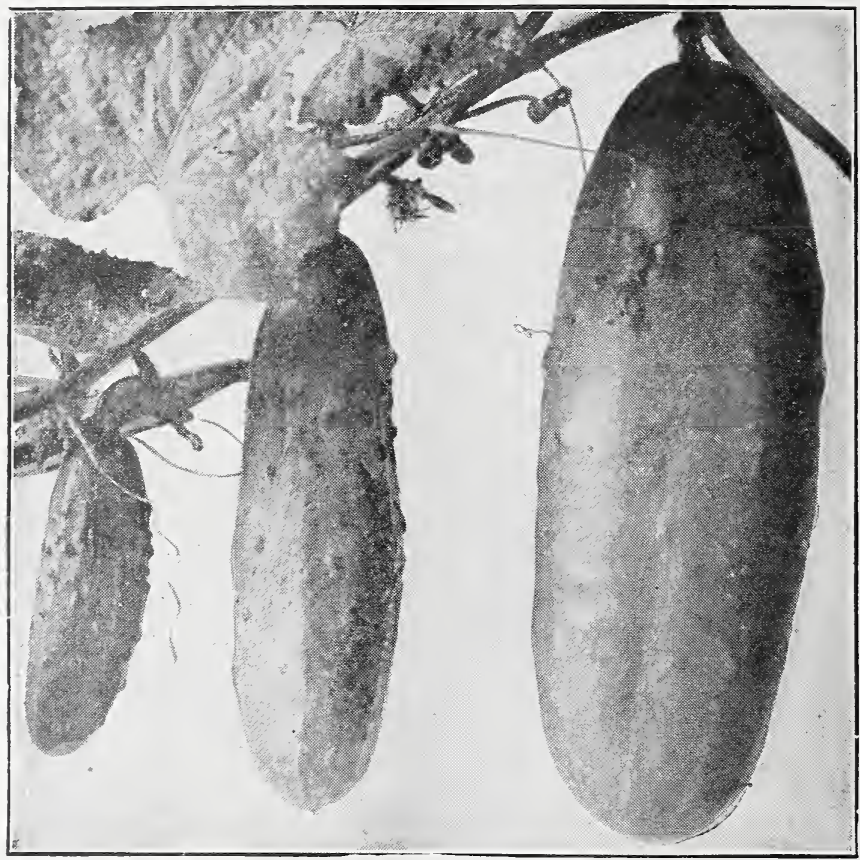

BOSTON PICKLING

\section{CUCUMBER}

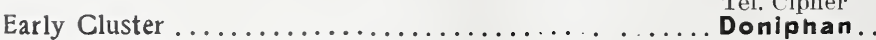

Early Short Green or Early Frame............... Dooly......

Snow's Pickling............................ Drew.......

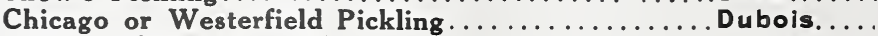

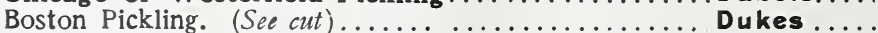

Jersey Pickling .......................... Dunklin....

Early White Spine.......................Dupage....

Arlington ............................. Durham....

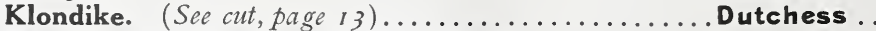

Extra Long or Evergreen White Spine........... Duval ......

Improved Long Green. We believe the stock we offer

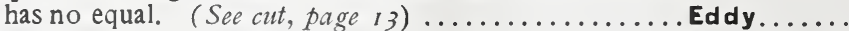

Small Gherkin (for pickles).................. Edmonson.

Per L b.

$\$ 045$ 
D. M. FERRY \& CO., DETROIT, MICH.

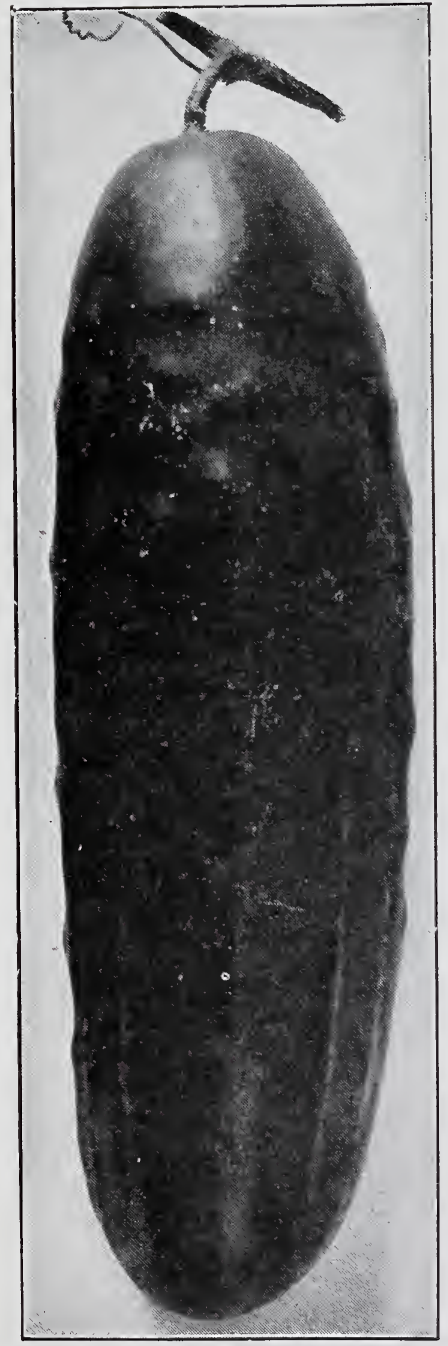

K ONCIKE

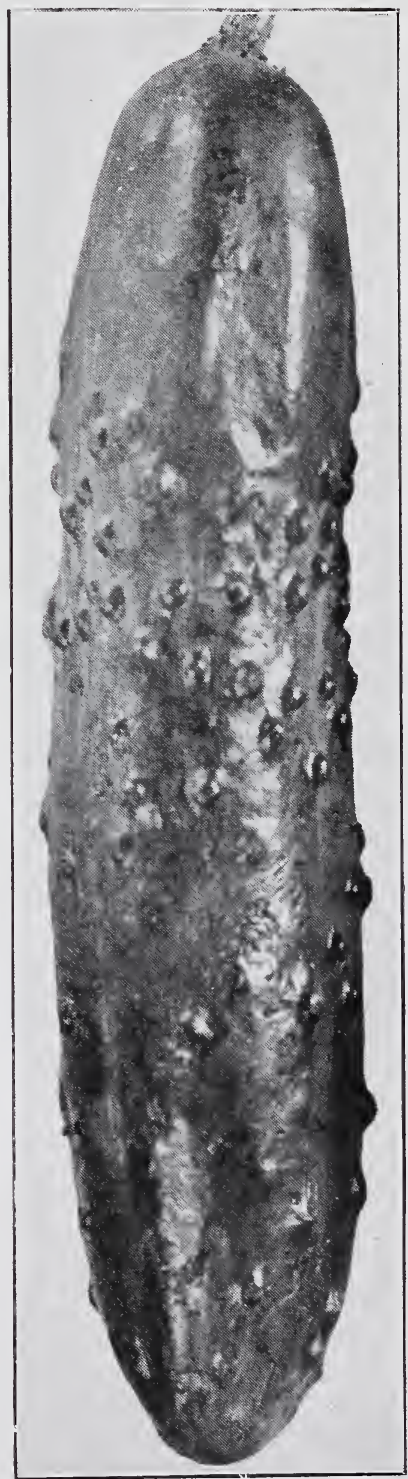

Improved Long Green 


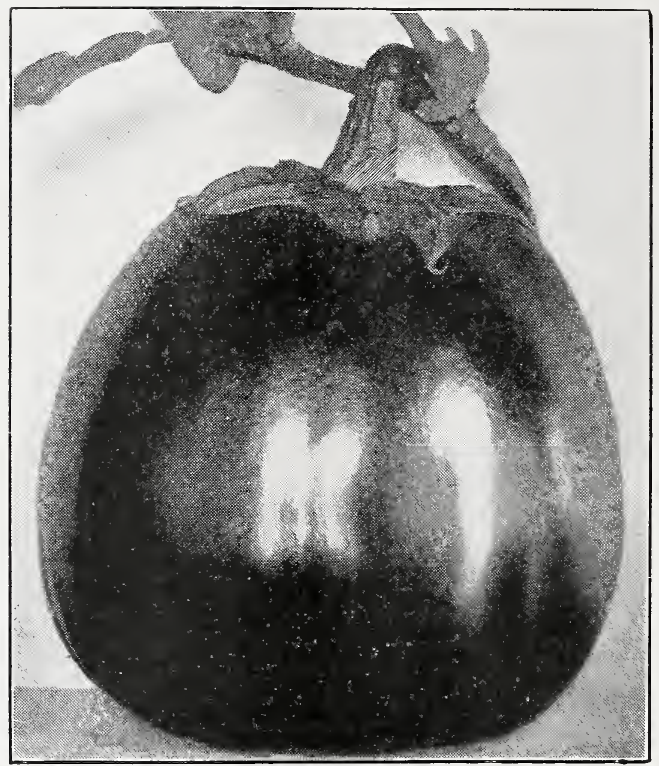

Ega plant, D. M. Ferry \& Co's improved large Purple

EGG PLANT

Tel. Cipher Per Lb.

Early Long Purple........................Elkhart... \$4 $\$ 40$

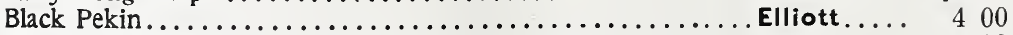

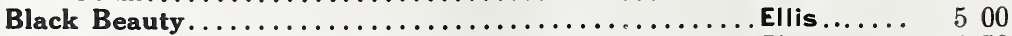

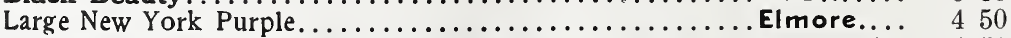

D. M. Ferry \& Co's Improved Large Purple, spineless.... Emanuel. . 450

\section{ENDIVE}

Large Green Curled........................ Emmet.... 85

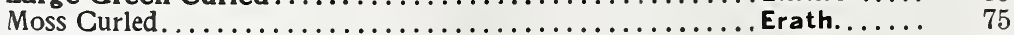

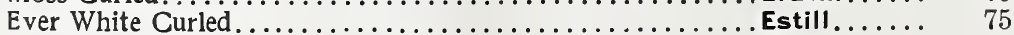

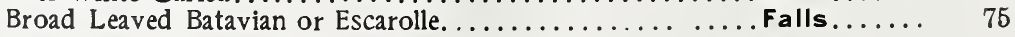

FENNEL

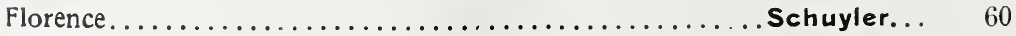

\section{GARLIC}

Bulbs

Faulkner... 
D. M. FERRY \& CO., DETROIT, MICH.

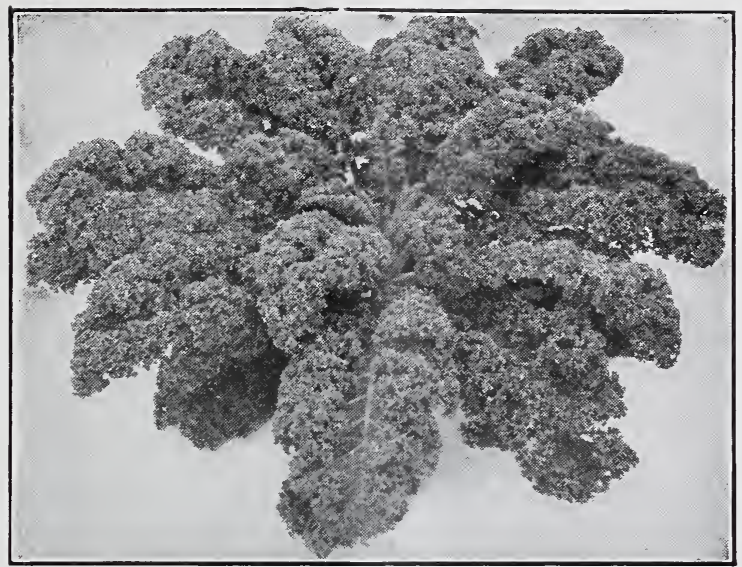

DWARF CURLED SCOTCH KALE

\section{KALE or Borecole}

Tel. Cipher Per Lb.

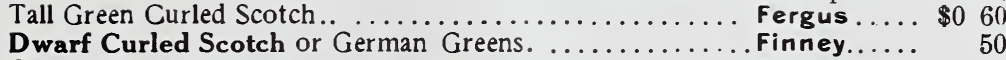

German Dwarf Purple............................. Fluvanna... 75

Siberian or Sprouts .. .......................... Fleming... 40

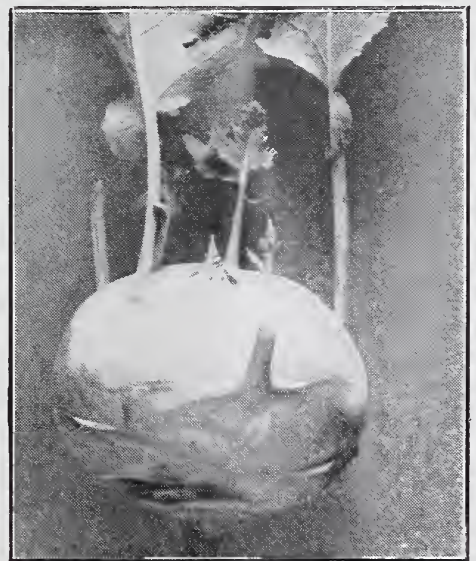

Kohl Rabi, EARLy White Vienna

KOHL RABI

Early White Vienna, extra for forcing............... Forest. Per Lb. Early Purple Vienna, very early................................... 125 Large Green ........................................ Franklin.... 75 


\section{LEEK}

London Flag. . Tel. Cipher Per Lb.

Large Ame

Large Rouen

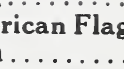

Fremont ... \$075

Monstrous Carentan.

Frio.........

Monstrous Carenta.

Gallatin.

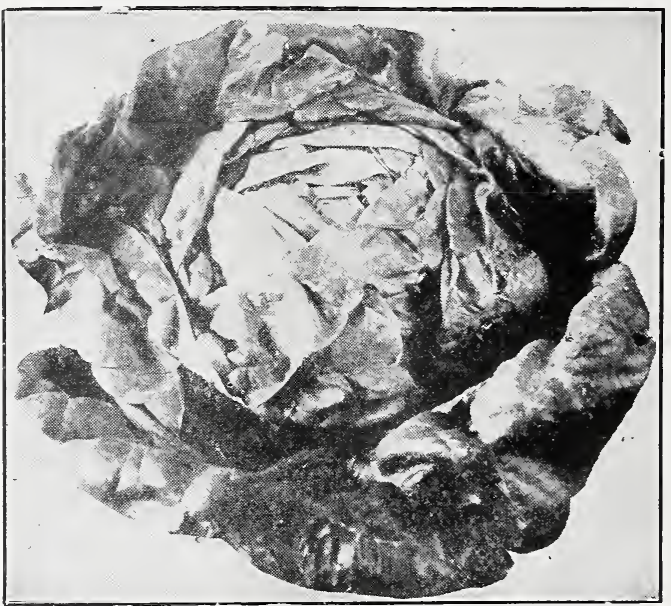

MAY KING

\section{LETTUCE}

May King, w. s. $($ See cut $) \ldots \ldots \ldots \ldots \ldots \ldots \ldots \ldots \ldots$ Garfield.... $\$ 065$

Early Tennis Ball, b. s. .......................Garvin...... 60

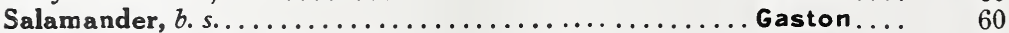

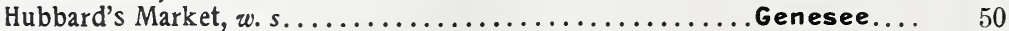

Mammoth Black Seeded Butter................Gentry..... 60

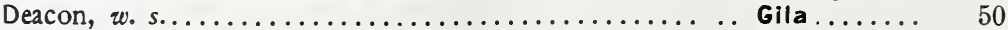

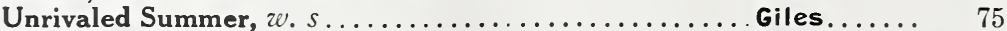

Big Boston, w. s.........................lliam.... 65

California Cream Butter or Royal Summer Cabbage, brown seed.Gilpin . . . . . 50

Jersey Winter, w.s..........................Glascock... 55

Brown Dutch, b. s. (verv hardy)............................... $\quad 55$

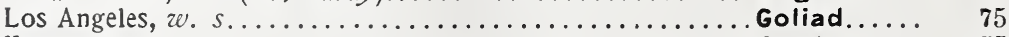

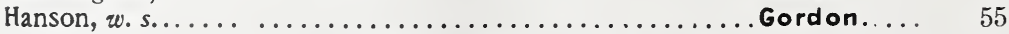

\section{CURLED OR LOOSE LEAVED VARIETIES}

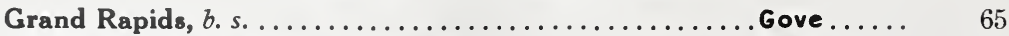

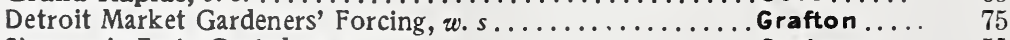

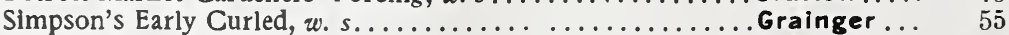

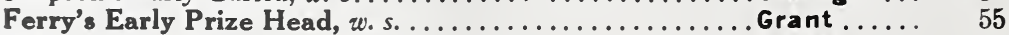

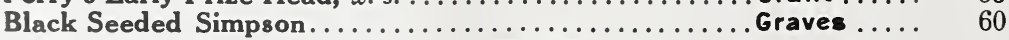

Early White Self-Folding Cos, w. s. (Romaine)........Gregg..... 75 


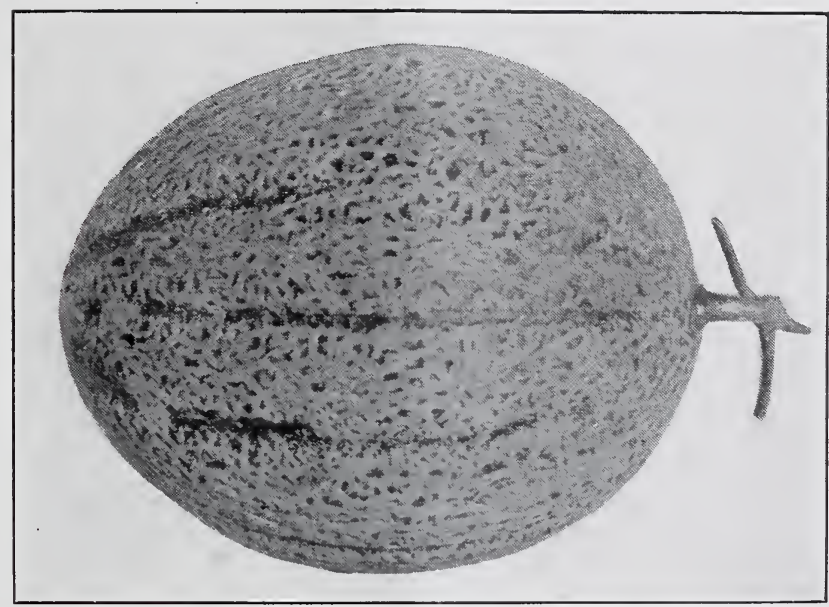

ADMIRAL TOGO

\section{MUSK MELON}

GREEN FLESHED VARIETIES

Tel. Cipher Per Lb.

Extra Early Citron $\ldots \ldots \ldots \ldots \ldots \ldots \ldots \ldots \ldots \ldots \ldots \ldots$ Griggs...... $\$ 045$

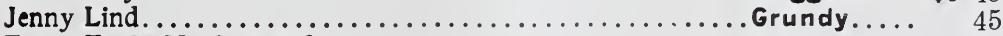

Extra Early Hackensack....................... Gunnison .. 50

Netted Gem or Rocky Ford ..................... Gwinnett... 45

Baltimore or Acme .................................... 45

Champion Market.................................... 50

Hackensack or Turk's Cap...........................nansford... 55

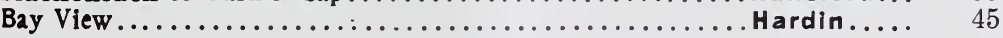

\section{ORANGE FLESHED VARIETIES}

Emerald Gem. For home use and near markets it leads all other kinds. on account of its sweetness and convenient size. Harford.... 60

Tip Top...................................... 55

Surprise....................................... 50

Extra Early Osage. Fully a week earlier than Osage, a little smaller, more nearly round and with more netting.........Hart...... 55

Petoskey or Paul Rose ............................... 50

Admiral Togo or Orange Fleshed Rocky Ford. Unexcelled in flavor. We consider this the best small shipping melon yet introduced. (Seecut) ................................

Hoodoo. A splendid orange fleshed variety of medium size. By many considered the finest shipping melon............ Hays...... 50

Defender. Originated and introduced by us in 1901. We believe it identical with Burrell's Gem ................. Heard ......

Osage or Miller's Cream. Our stock is unsurpassed....... Hennepin ..

CASSABA MELON, Golden Beauty................. Hertford.. 100 


\section{WATER MELON}

Tel. Cipher Per Lb.

Harris' Earliest .......................... Hidalgo... \$0 40

D. M. Ferry \& Co's Peerless (Ice Cream) ...........Hocking.... 35

Round Light Icing ................................. 35

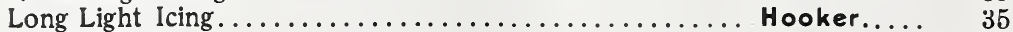

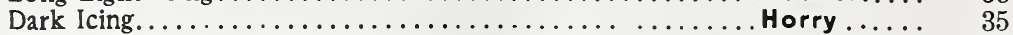

Monte Cristo or Kleckley's Sweets. (See cut)........... Houston... 50

Florida Favorite ................................... 35

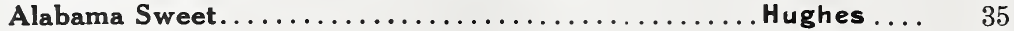

Tom Watson......................................... 45

Ferry's Iceberg.......................... Humboldt. . 25

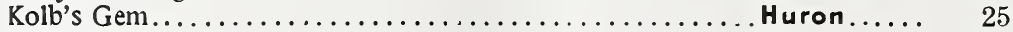

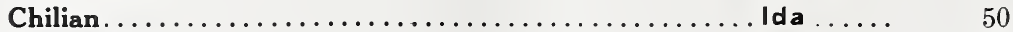

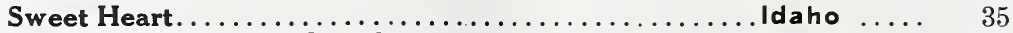

Gypsy or Georgia Rattlesnake................. Ingham ... 35

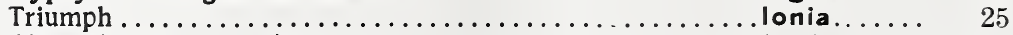

Citron (for preserving).......................... 50

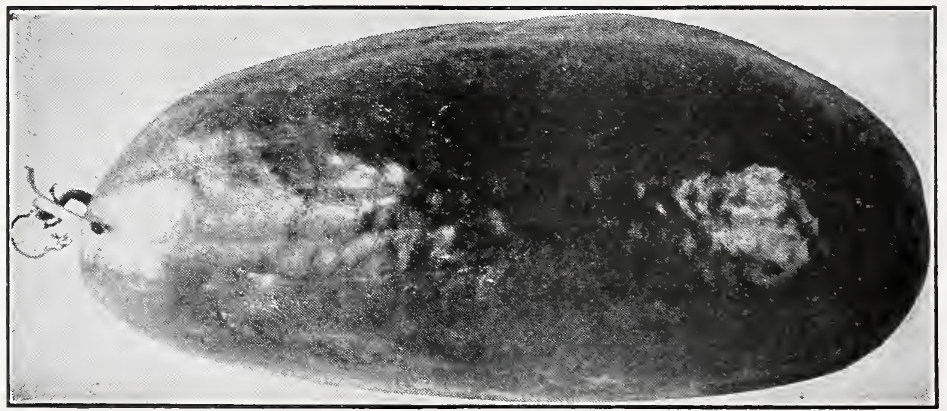

Monte Cristo or KLeCKLey's SWEets

MUSHROOM SPAWN Tel. Cipher Per Brick

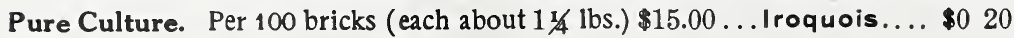

MUSTARD

White English. Isabella....\$ \$0 30

Southern Giant Curled.... ......................

Large Smooth Leaved

Is anti..... 35

\section{NASTURTIUM}

Tall Mixed Garden. (See other Nasturtiums, page 40)......... Itasca......

\section{OKRA or Gumbo}

White Velvet..........................Jackson... 20

Perkins' Mammoth Long Pod.....................Jasper..... 20

Dwarf White............................Jenkins.... 20 


\section{ONION}

Our onion seeds are grown from the choicest selected bulbs raised from pedigree seed of the choicest selected types. You may find onion seed offered for less than ours. You cannot find any of better quality, no matter how high the prices asked.

Extra Early Red

Tel. Cipher Per Lb.

.................. \$1 50

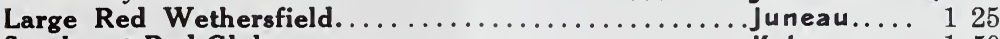

Southport Red Globe.........................Kalawao ... 150

Large Yellow Dutch or Strasburg......................Kanabec... 125

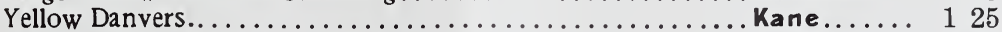

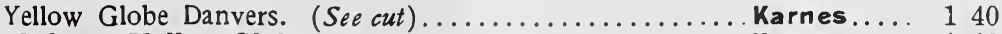

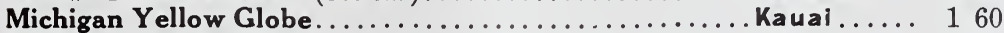

Southport Yellow Globe................................... 160

Mammoth Yellow Spanish or Prizetaker ..............Kennebec.. 150

White Portugal (American Silverskin) ................Kenton.... 250

White Globe..............................Kern ...... 300

Extra Early Barletta........................Keokuk .... 300

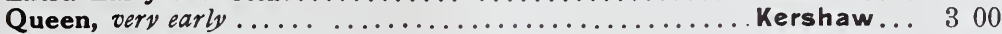

Early Neapolitan Marzajola ..........................Keweenaw . 250

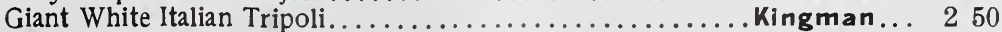

Mammoth Silver King............................. Kiowa..... 250

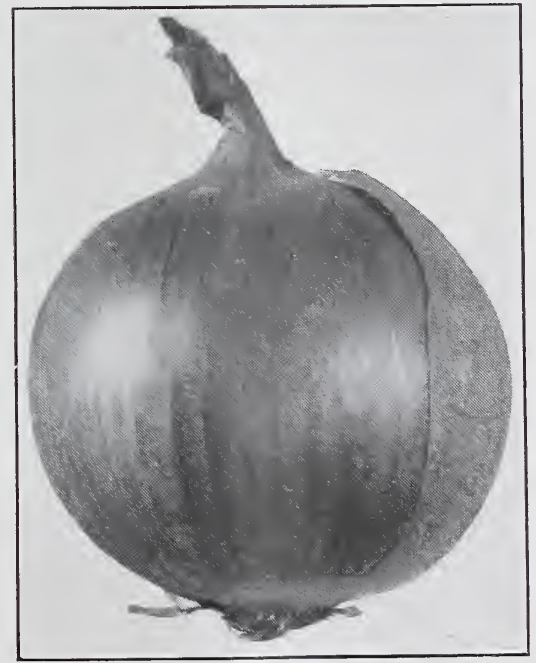

Yellow Globe DanVERS

PARSLEY

Plain

Tel. Cipher Per Lb.

Kittitas.....\$0 35

Champion Moss Curled ....................Klamath ... 35

Hamburg or Rooted. ............................. 45

\section{PARSNIP}

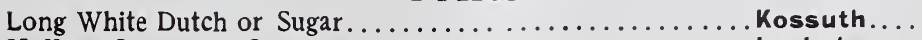

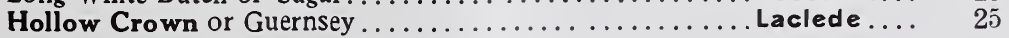


D. M. FERRY \& CO., DETROIT, MICH.

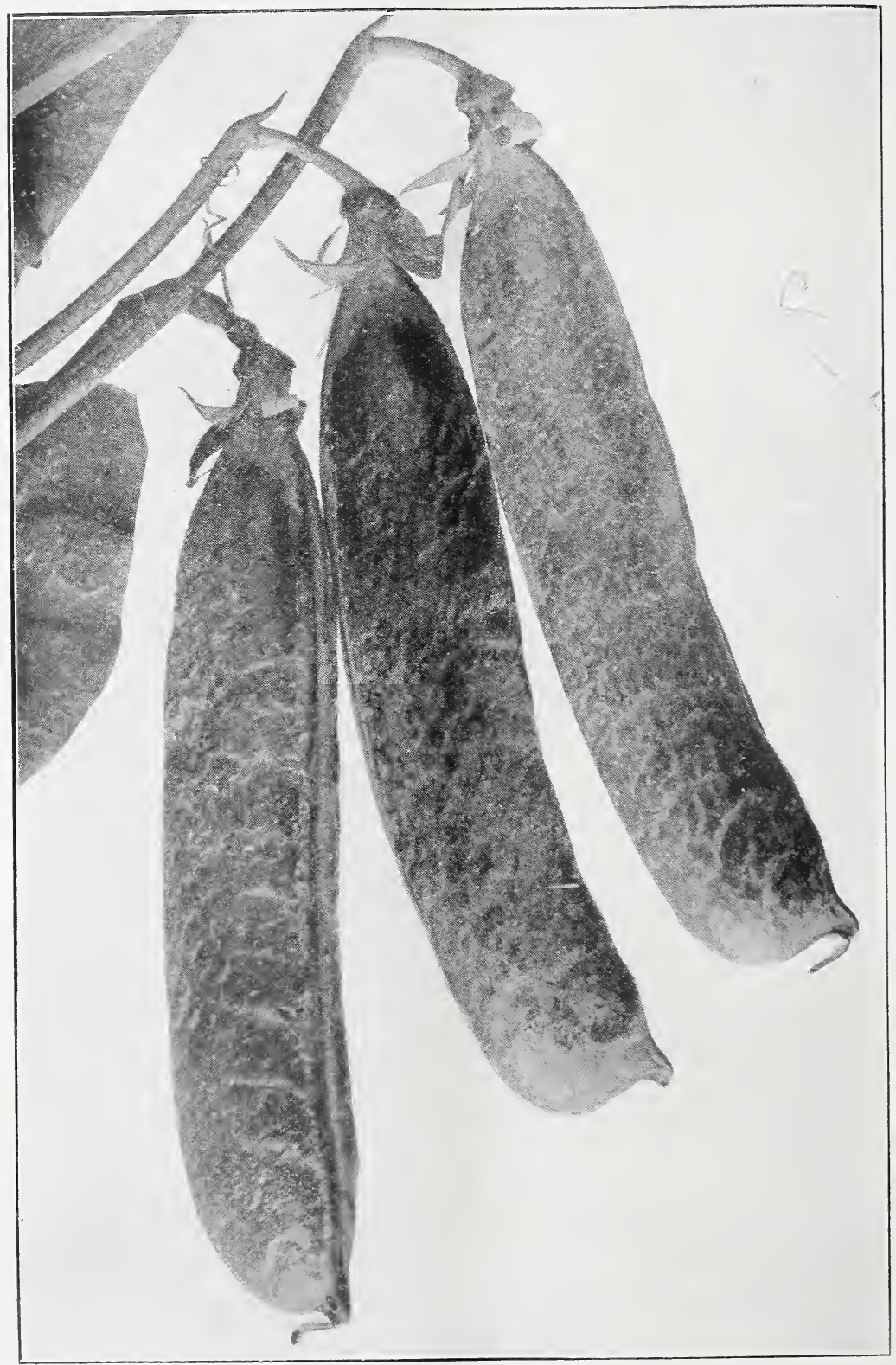




\section{PEAS}

Not less than 10 lbs. at $100 \mathrm{lb}$. rate. Wrinkled varieties marked thus*.

FIRST EARLY AND EARLY SORTS

Tel. Cipher Per Lb. 100 Ler

Ferry's First and Best.

Lagrange..\$0 $15 \$ 1000$

Earliest of All or Alaska......................... 20

1500

*Thomas Laxton. (See cut, page 20) ............Lamoille... 151100

*Gradus............................................................ 1200

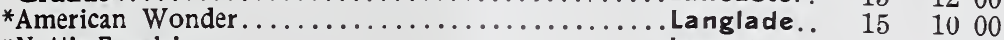

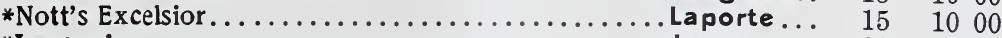

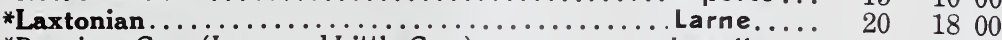

*Premium Gem (Improved Little Gem)............. Lasalle.... $15 \quad 1000$

*Little Marvel.................................... $15 \quad 1200$

SECOND EARLY SORTS

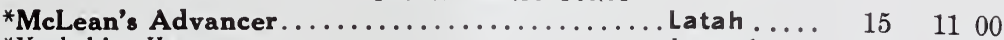

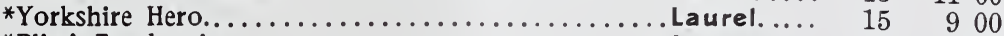

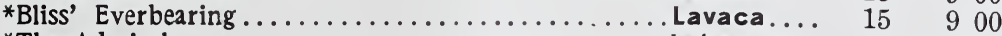

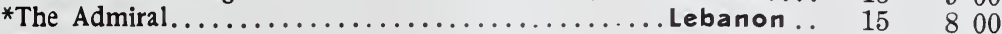

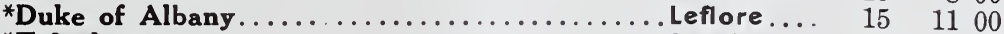

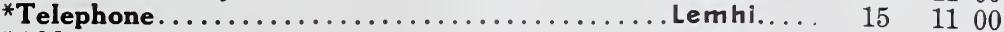

*Alderman ................................. 151100

LATER SORTS

*Improved Stratagem.................... Letcher... $15 \quad 1200$

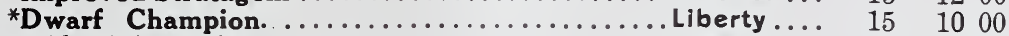

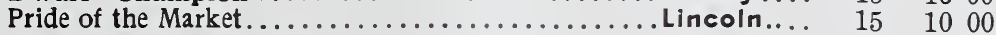

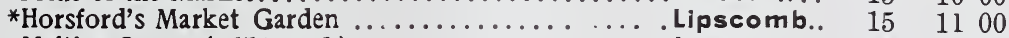

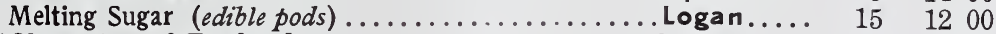

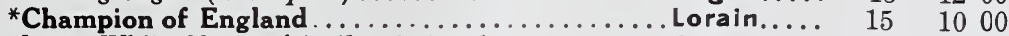

Large White Marrowfat (band-picked)............Luisa.... $15 \quad 1000$

Large White Marrowfat.......................... sove sold out

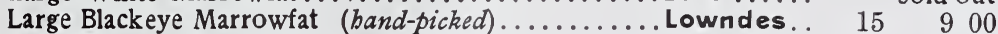

Large Blackeye Marrowfat..................ucas.... $15 \quad 850$

FIELD SORTS

Common White................................... market

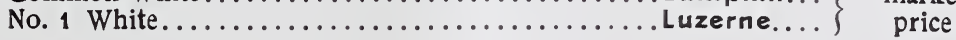

PEPPER

Per Lb.

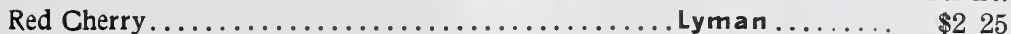

Red Chili............................................ 225

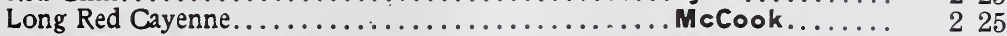

Large Bell or Bull Nose ................................. 250

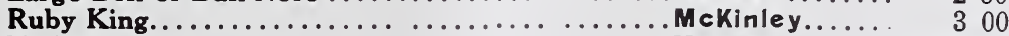

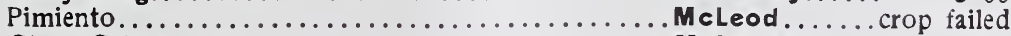

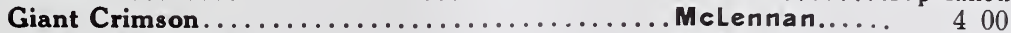

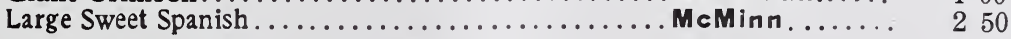

PUMPKIN

Per Lb. $100 \mathrm{Lbs}$.

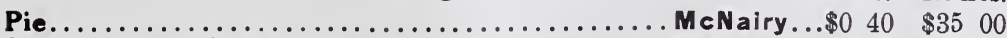

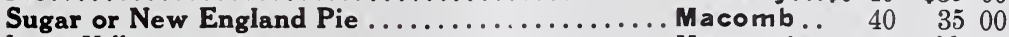

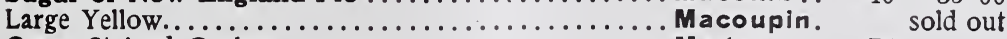

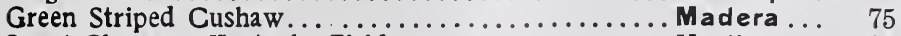

Sweet Cheese or Kentucky Field .......................... $35 \quad 3000$ 


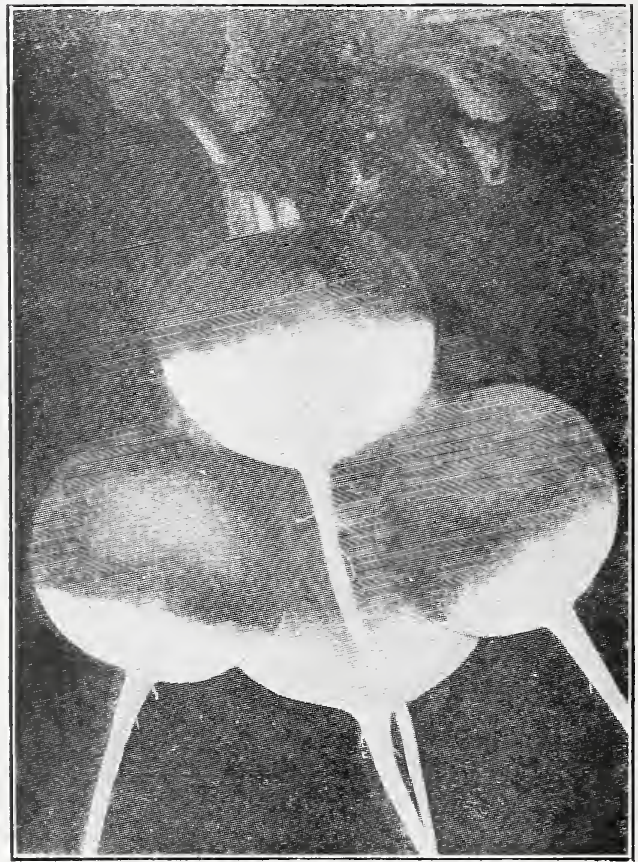

EARLy SCARLET TURNIP, White TIPPED

RADISH

Tel. Cipher Per Lb.

Non Plus Ultra or Early Deep Scarlet Turnip, Forcing ...... Malheur . . \$0 $\$ 5$

Prussian Globe..............................Mahaska... 35

Early Scarlet Globe.........................Manistee... 40

Early Scarlet Turnip, White Tipped, Forcing........... Marengo... 35

Early Deep Scarlet, Olive Shaped, Short Leaf ............ Maries..... 35

Early White Turnip.............................. 40

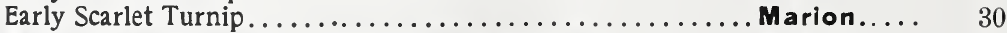

Early Scarlet Turnip, White Tipped. (See cut).........Marlboro... 40

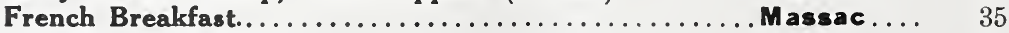

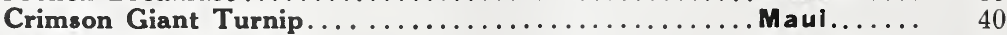

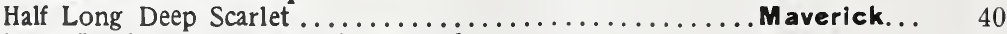

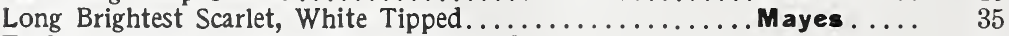

Early Long Scarlet, Short Top,Improved. . . . . . . . . . . Meagher... 30

Wood's Early Frame....................................... 30

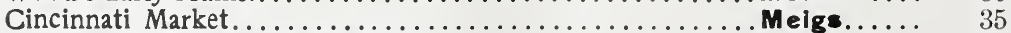

Improved Chartier or Shepherd .................Menifee.... 35

Early Golden Yellow Oval.............................. 40

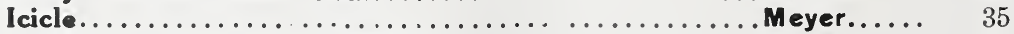

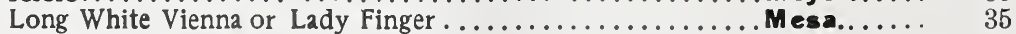

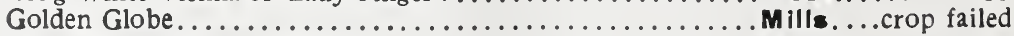

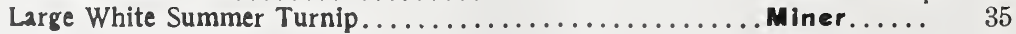

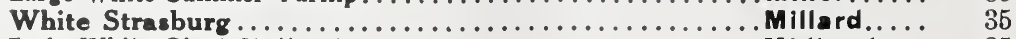

Early White Giant Stuttgart....................... 35 


\section{RADISH-Continued}

\section{WINTER VARIETIES}

Tel. Cipher Per Lb.

Scarlet China. (See cut $) \ldots \ldots \ldots \ldots \ldots \ldots \ldots \ldots$.................. \$0 35

Celestial. Roots long, cylindrical with beautiful white skin and flesh; quality excellent. $($ See cut $) \ldots \ldots \ldots . . . \ldots$............. 40

California Mammoth White China.......................... 40

Round Black Spanish..........................Monroe.... 40

Half Long Black Winter. Roots half long, grayish black;

flesh white, crisp, pungent and well flavored.........Montcalm.. 40

Long Black Spanish............................ntour... 40

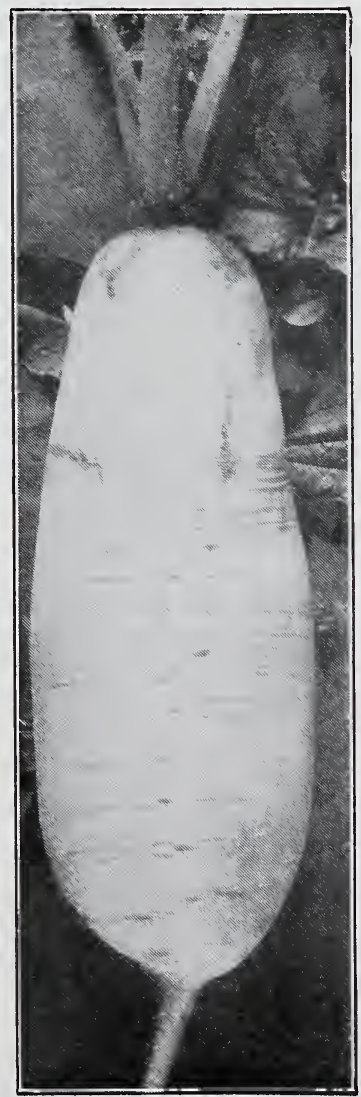

Celestial

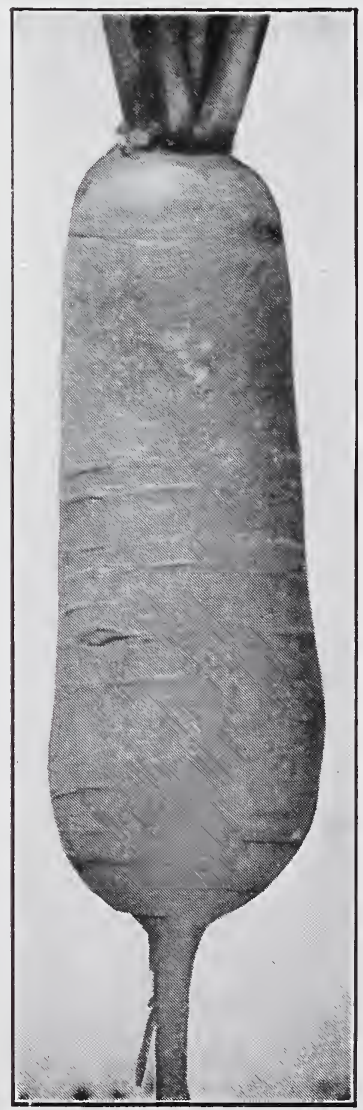

SCARLET CHINA 


\section{RHUBARB}

Tel. Cipher Per Lb.

Rhubarb or Pie Plant.............................. \$0 60

" " " " roots.......per doz., $\$ 1.00 \ldots . \ldots$. Morgan

\section{SALSIFY or Vegetable Oyster}

Mammoth Sandwich Island............................

\section{SORREL}

Large Leaved French...................... Moultrie... 125

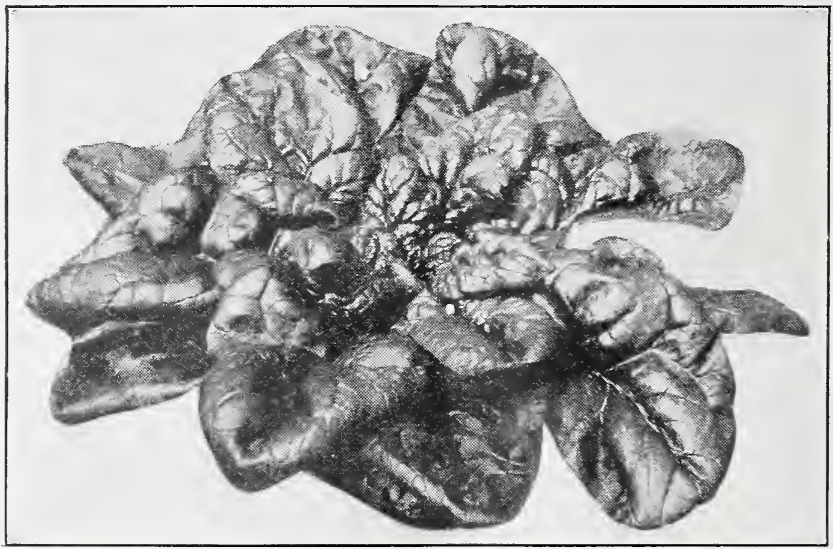

VICTORIA

\section{SPINACH}

Savoy Leaved or Bloomsdale Broad Flanders.................. ........ Nance...\$0 $60 \$ 5000$ Improved Thick Leaved (Monstrous Viroflav)...... Natrona... $60 \quad 6000$ Early Giant Thick Leaved (very short crop) ...... Nash ..... sold out Victoria. (See cut) ...................... Navarro... $70 \quad 6000$

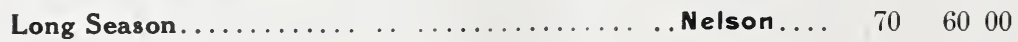
Long Standing.................................. $60 \quad 5000$ Long Standing Prickly.................Neshoba... sold out

New Zealand (Tetragonia) ................. Newberry 50 


\section{SQUASH}

SUMMER SORTS

Tel. Cipher Per Lb.

Long Island White Bush..................... Noble.....\$0 65

Early White Bush Scallop................................... 60

Mammoth White Bush Scallop................... Newton .... 65

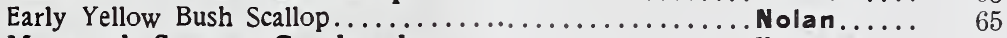

Mammoth Summer Crookneck................. Nowata.... 75

Summer Crookneck. .......................... Nuckolls... 70

Italian Vegetable Marrow (Cocozella di Napoli)............ Nye...... 60

\section{AUTUMN AND WINTER SORTS}

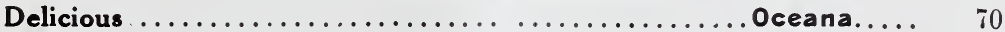

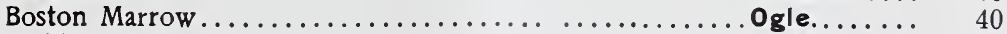

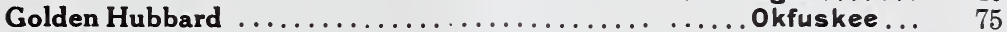

Hubbard. (See cut).................................. 75

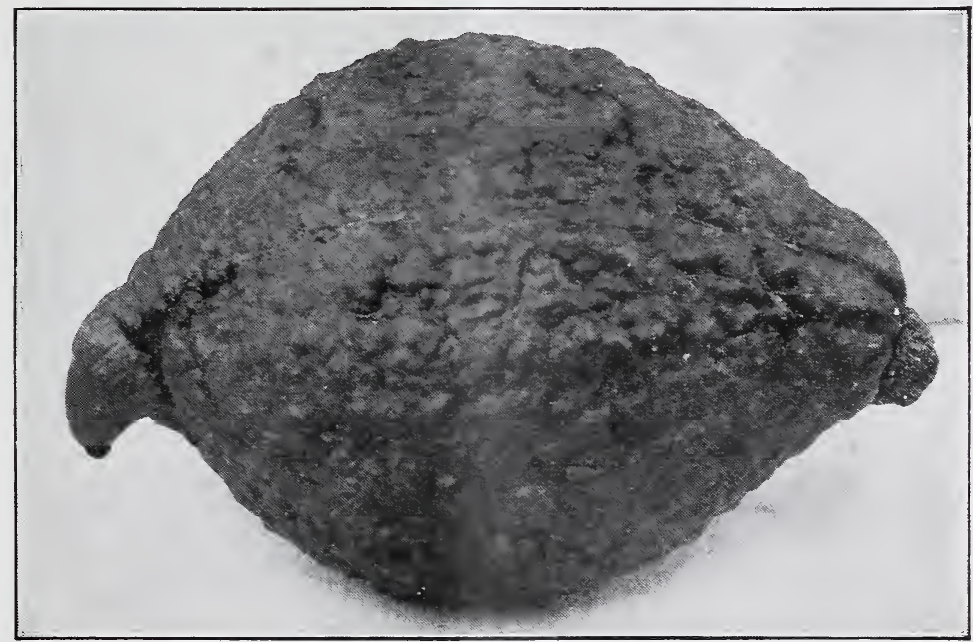

HUBBARD

TOBACCO

Sterling. ............................ Oneida...\$0 $15 \$ 150$

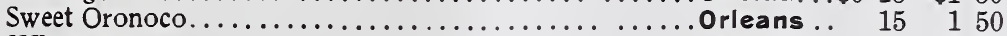

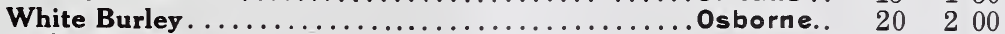

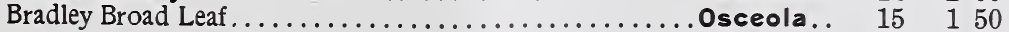

Persian Rose ........................................ 2020

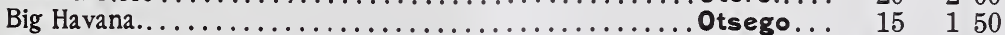

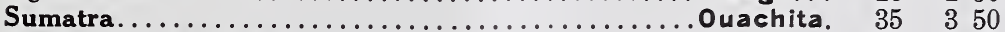

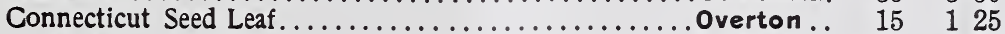

Havana .............................. Owsley... $25 \quad 225$ 


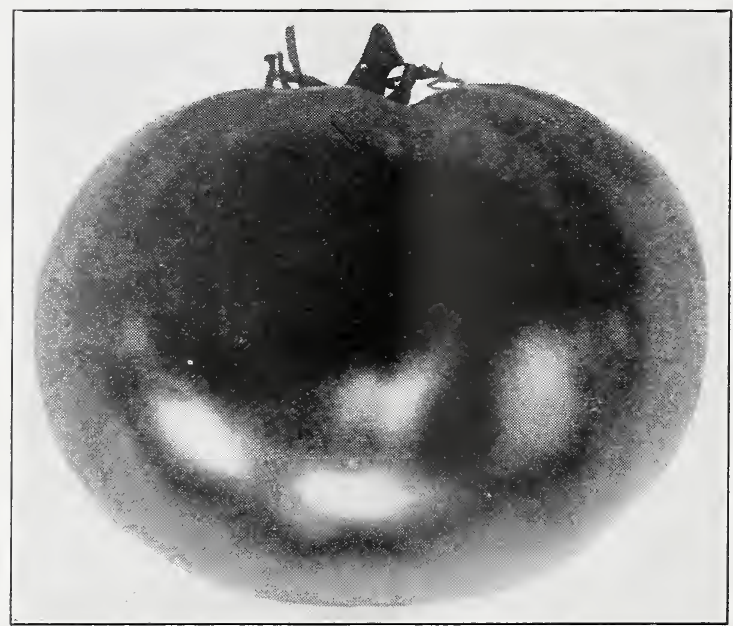

JOHN BAER

\section{TOMATO}

Earliana. A particularly profitable first early scarlet tomato for market gardeners .......................... 0xford... $\$ 250$

John Baer. Extra early, scarlet fruited, hardy and productive. Shippers should try it. $($ See cut $) \ldots . . . \ldots \ldots \ldots \ldots$............ 400 June Pink. Similar to Earliana but purplish pink........ Ozaukee ... 225 Bonny Best ...............................Panola..... 250

Chalk's Early Jewel. Considered by many to be the best all around tomato for the home garden. Early, of large size, smooth and of excellent quality ..............Page..... 250

Early Detroit. The largest and best of the early, purplish pink tomatoes; larger than Acme and earlier maturing than Beauty; an exceptionally valuable addition to this class. A splendid shipper ....................... Pacific .... 300

Acme. Known also as Essex Early Hybrid.............Pamlico... 200

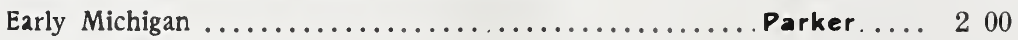

Livingston's Globe.........................Patrick .... 300

Beauty. A larger, smoother fruit than the original stock and one of the smoothest and best of the large purplish pink sorts...................................... 200 


\section{TOMATO-Continued}

Improved Trophy. Our carefully developed stock is notably Tel. Cipher Yer Lb. superior in size, interior color and regularity of fruit.....Pemiscot... \$2 25

Perfection .................................Pecos..... 200

Stone. One of the largest and most solid, main crop or late, bright red varieties. Our stock is distinctly superior to most of that offered under this name; being more uniform, better colored and larger. (See cut $) \ldots \ldots \ldots \ldots \ldots \ldots$.............. 200

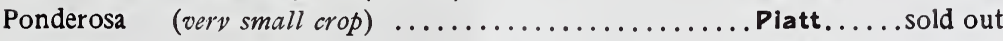

Matchless...................................Person..... 225

Coreless .......................................... 225

Dwarf Stone ............................. Pike....... 250

Dwarf Champion ...........................Pinal...... 250

Peach.......................................Plute...... 175

Golden Queen...............................Platte..... 175

White Apple..............................Plymouth .. 175

Red Pear................................Polk...... 175

Yellow Pear....................................Pontotoc... 175

Yellow Plum .................................Portage.... 175

Red Cherry ................................Posey..... 175

Yellow Cherry.................................Powell..... 175

Yellow Husk or Ground Cherry.... ...............Prairie .... 150

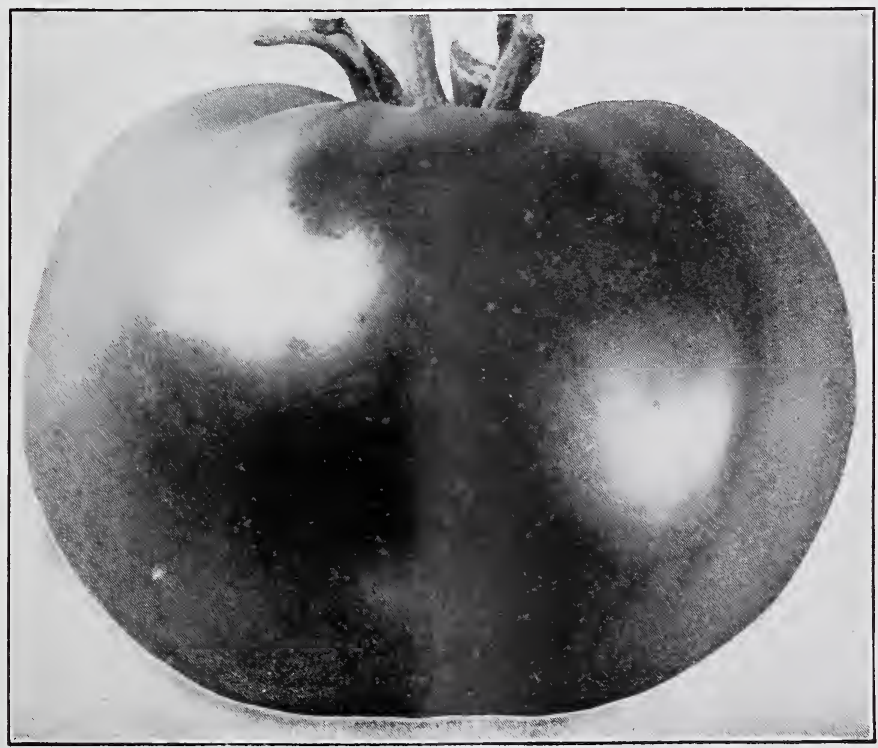

STONE 


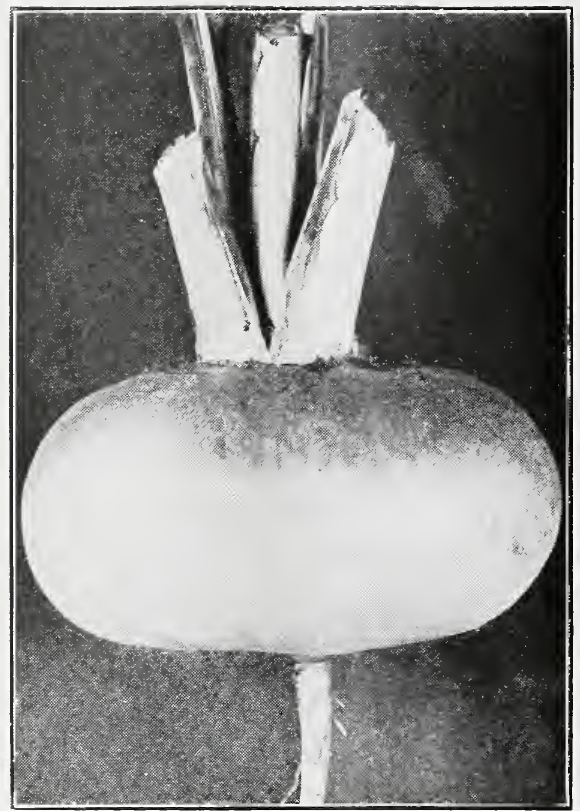

Early Purple Top Strap leaved

\section{TURNIP}

WHITE FLESHED SORTS

Tel. Cipher Per Lb.

Extra Early White Milan.

Preble.

Extra Early Purple Top Milan. Roots purple-red on upper porition, white below, smoo:h, flat; flesh mild, sweet and tender. Well adapted for forcing..................Presidio.... 100

White Egg. An early, oval or egg-shaped variety with smooth, clear white roots of medium size; flesh fine grained and sweet...................................Price......

Early White Flat Dutch, Strap Leaved. A most excellent early garden variety. Roots medium sized, flat; color white. Very early; sweet and tender. It is desirable for table use.............................. Pueblo.....

Early Purple Top, Strap Leaved. Very extensively used for the table. Roots flat, of medium size, purple or dark red above ground, white below. Flesh white, fine grained

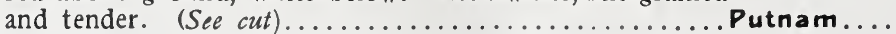

Purple Top White Globe. The roots are of excellent quality and are suitable for the table as well as for stock feeding. 


\section{TURNIP-Continued}

WHITE FLESHED SORTS

Cow Horn or Long White. Roots long, often twelve to Tel. Cipher Per Lb. fifteen inches; flesh fine grained and well flavored........Rabun.....\$0 40

Large White Norfolk.. ....................Rains..... 30

Pomeranean White Globe....................Ralls...... 30

Sweet German. An excellent, late maturing strap leaved sort, also sold as Sweet German Ruta Baga. The roots are medium large, somewhat globular, solid and sweet.......Randall... 35

Seven Top, for turnip greens. . . ..................Ransom.... 30

Southern Prize.......................Ravalli.... 35

YELLOW FLESHED SORTS

Large Amber Globe. Roots large, globular; skin clear yellow except the top which is tinged with green. Flesh light

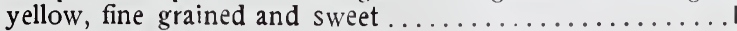

Yellow Globe.............................Redwood...

Yellow Stone. Roots of medium size, globular; skin smooth, attractive pale yellow. Flesh crisp, very tender and of fine

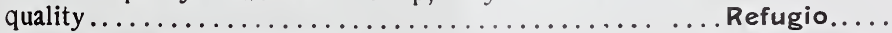

Orange Jelly or Golden Ball ...................Renville.... 30

Purple Top Yellow Aberdeen.................... Rhea...... 30

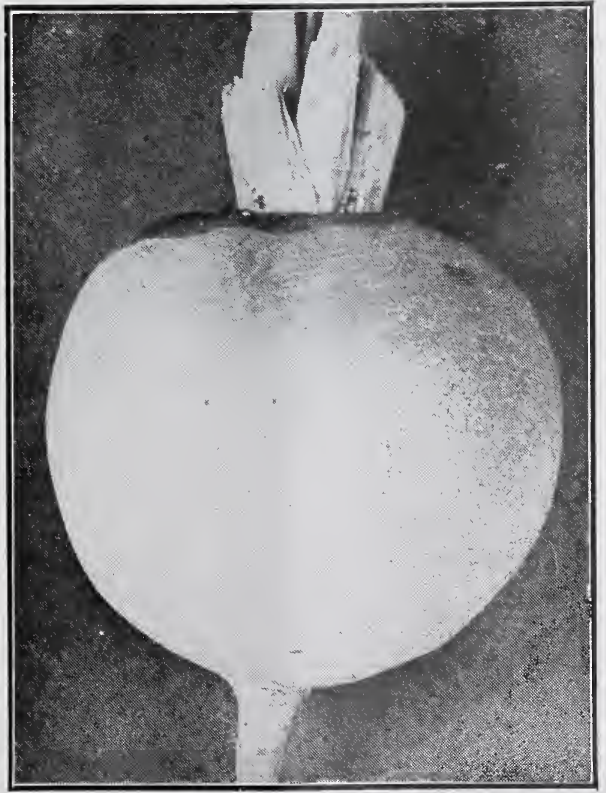

Purfle Top White Globe 


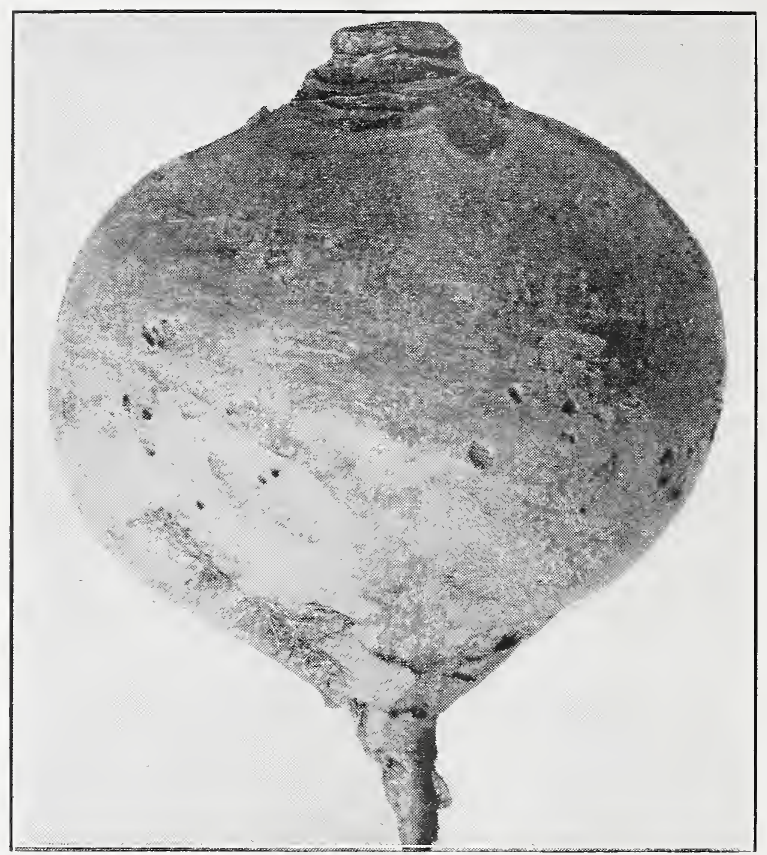

purple top Yellow ruta baga

\section{RUTA BAGA OR SWEDE}

Tel. Cipher

American Purple Top or Improved Long Island. . Richmond...... . . \$0 40 Bangholm Swede........................Rolette......... 35

Carter's Imperial Purple Top. . . . . . . . . . . . . Ritchie......... 35

D. M. Ferry \& Co's Improved Purple Top Yellow. Roanoke...... . . 35

Drummond's........................... Rockdale....... 35

Hall's Westbury........................Roseau......... 40

Hartley's Bronze Top......................Rowan......... 40

Laing's Purple Top. Strap leaved..............Robeson ........ 40

Monarch or Tankard .............................. 40

Perfection White. Absolutely neckless..........Sabine........ 45

Skirving's King of Swedes..................Ross.......... 35

Sutton's Champion.....................Rush.......... 35

Universal or Canadian Gem..............Riley......... 40

White or Sweet Russian...................Russell......... 35

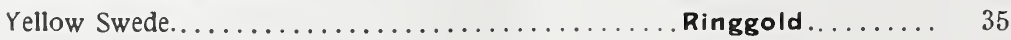




\section{AROMATIC, MEDICINAL AND POT HERBS}

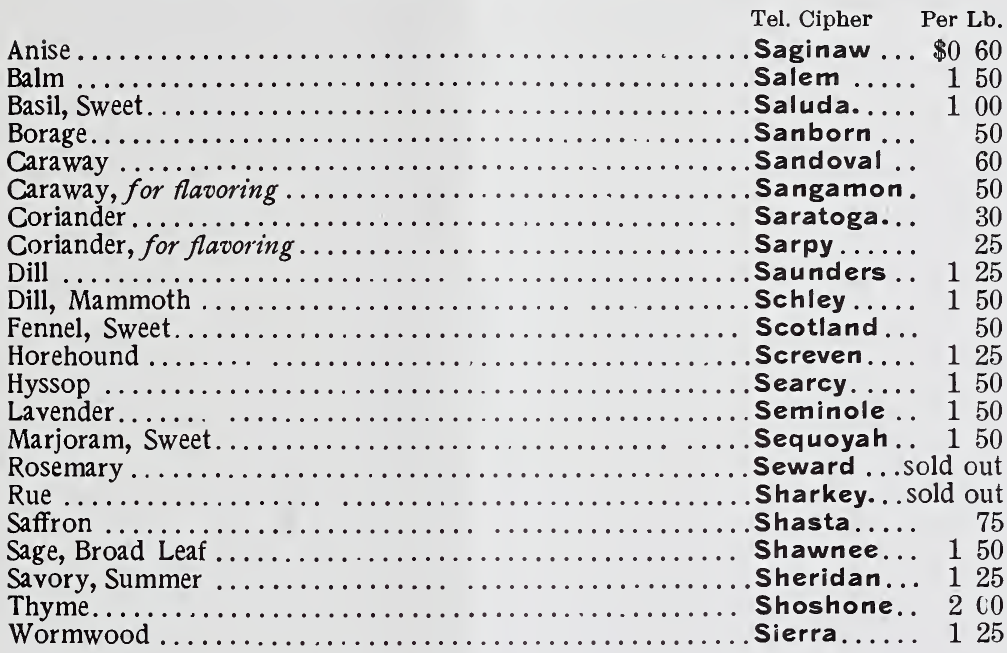

\section{GRASS SEED}

Prices are subject to fluctuations of the market. Not less than $1 / 4 \mathrm{lb}$. at pound rates nor less than 25 lbs. at $100 \mathrm{lb}$. rate. Terms net cash ten days.

Awnless Brome Grass. Sonoma.. Market price

Canadian Blue Grass. . Stone...

Creeping Bent Grass

Skagit....

Hard Fescue Spokane.

Kentucky Blue Grass, fancy clean; choicest grade...... Stewart.. Orchard Grass, choice ........................ Spencer.. Perennial Rye Grass Stearns. Red Top, Fancy (cleaned from chaff) ................ Snyder.. Red or Creeping Fescue ....................... Stafford.. Rhode lsland Bent Grass ......................... Sioux.... Sheep's Fescue ........................... Stanton..

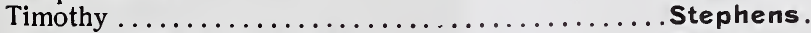

Westerwold Italian Rye Grass................... Starr.... Wood Meadow Grass Steuben. .

Extra Fine Mixed Lawn Grass (Central Park Mixture).

Our Central Park Mixture is the best possible for

permanent lawns...

Stoddard. $\$ 035 \$ 3000$

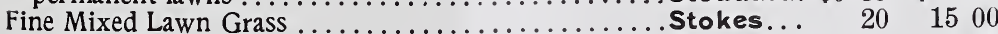

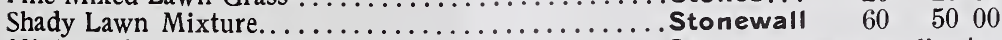

Mixtures for Golf Links................... Story . . Prices on application 


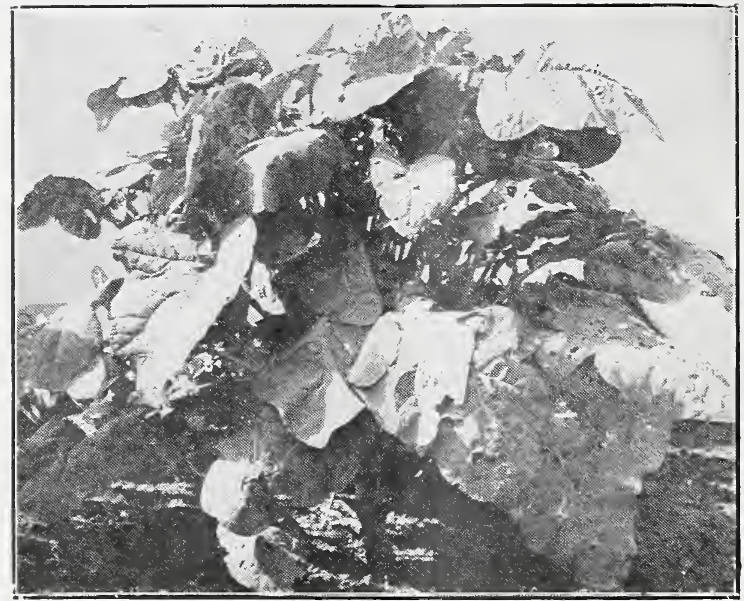

DWARF Es8EX RAPE

\section{CLOVER} Alsike or Lucerne. Choicest American grown... Stutsman .

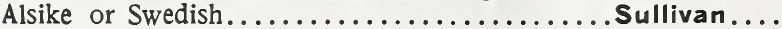
Mammoth or Large Red ................. Summers... Medium Red or June ......................... White Dutch, choice.

Sumner.... Market price

$\begin{array}{ll}66 & 66 \\ 66 & 66 \\ 66 & 66 \\ 66 & 16\end{array}$

\section{MISCELLANEOUS FARM SEEDS}

Barley, Common...................... Suwanee... Buckwheat, Japanese.....................Talbot...... Silver Hull....................Taney......

Flax Seed, for sowing......................... Tarrant..... Hungarian ..........................Tattnall.... Millet, Common........................Tazewell ... "German or Golden....................... Telfair...... Rye, Spring.......................... Tensas.... "Fall or Winter...................... Terrell..... Rape, Dwarf Essex, for sowing. (See cut).........Terry......\$0 10 Sunflower, Large Russian .................Tioga ..... 10 Sorghum (Sugar Cane) Albaugh Early.......... Texas...... 10

Vetch or Tare, Spring...................Tipton..... 10 Market price " Hairy or Sand (Winter) .............. Todd...... 20

\section{BIRD SEEDS}

Canary, recleaned.......................Tompkins.. 10

Hemp ..............................Toombs... 10

Lettuce............................. Towner... 15

Maw .......................................... 60

Millet...................................... 10

Rape............................Trigg...... 10

Mixed Bird Seed.................... Trinity .... 10 


\section{A S E LE C T E D L IS T}

\section{E \\ FLOWER SEEDS IN BULK}

We will not sell less than $1 \mathrm{oz}$. of sorts priced at $20 \mathrm{c}$. per oz. or under but we will put up $1 / 2 \mathrm{oz}$. of seeds priced at $25 \mathrm{c}$. per oz. and over; $1 / 4 \mathrm{oz}$. of seeds priced at $75 \mathrm{c}$. per oz. and over; $1 / 8 \mathrm{Oz}$. of seeds priced at $\$ 2.00$ per oz. and over.

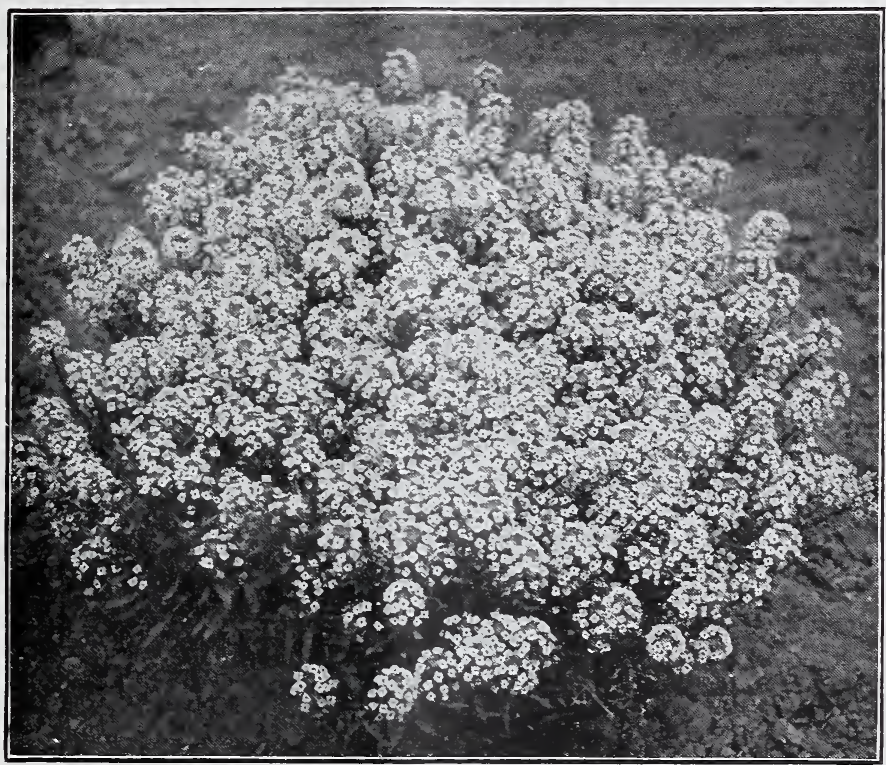

ALySSUM

Per Oz. Per Lb.

Acroclinium, Album................................ \$0 $10 \$ 100$

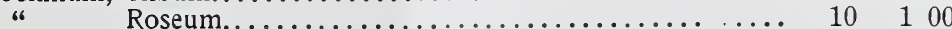

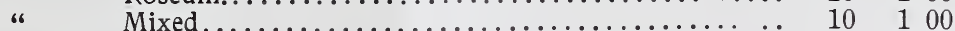

Ageratum Mexicanum.............................. $15 \quad 150$

“ Tom Thumb, dwarf blue........................ 30 . 30 . 300

“ Imperial, dwarf white.......................... $30 \quad 300$

Alyssum, Sweet (Maritimum).......................... $15 \quad 125$

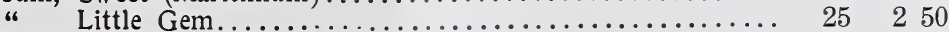

Amaranthus tricolor (Joseph's Coat).................... 20200

Asparagus sprengeri............................ 40

Aster, Queen of the Market, Lavender .................. 60

" " " " " Light Blue..................... 60

" " ، " " "

، " " " " "

، " " " " "

، " " " " 
Aster, Victoria, Mixed ............................. $\$ 100 \$ 1000$

Per Oz. Per Lb.

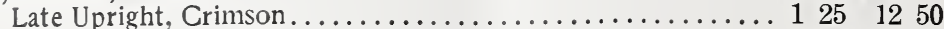

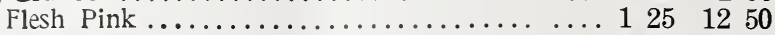

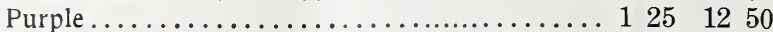

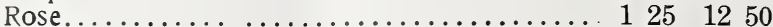

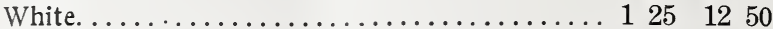

Mixed.......................... 1251250

Truffaut's Perfection Peony Flowered, Mixed............ $85 \quad 850$

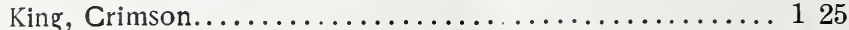

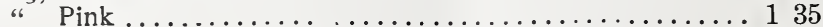

" Violet ............................ 135

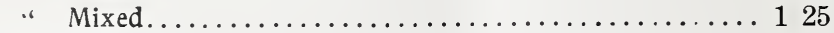

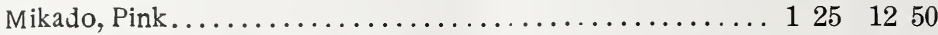

Giant Crego or Comet, Crego Pink............... 1251250

Crimson.................. $125 \quad 1250$

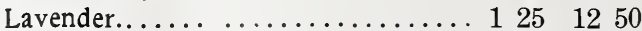

Light Blue................ $125 \quad 1250$

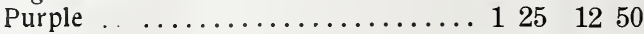

Rose ................... $125 \quad 1250$

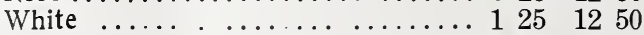

Mixed ................... $100 \quad 900$

Ferry's Late Branching, Azure Blue.................. 80800

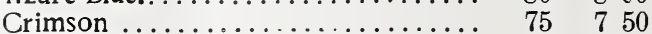

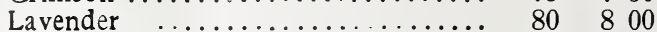

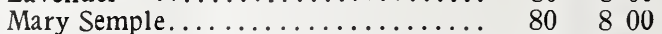

Peerless Pink................. 100

Purple ................... $80 \quad 860$

Rose Pink ............... $80 \quad 800$

Tyrian Rose.................. $80 \quad 800$

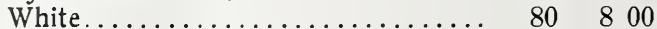

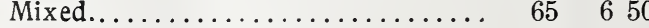

$75 \quad 750$

Balloon Vine (Cardiospermum halicacabum)................ $15 \quad 100$

Balsam, Double or Camellia Flowered, Pure White............. $40 \quad 400$

Solferino............ $40 \quad 400$

Extra Fine Mixed.......... $40 \quad 400$

Dwart Mixed........................ $25 \quad 250$

Tall Mixed. .............. $20 \quad 200$

Calliopsis (Coreopsis tinctoria) fine mixed ................ $10 \quad 80$

(Lanceolata grandiflora) ..................... $60 \quad 600$

Canary Bird Flower (Tropæolum canariense)................ $15 \quad 150$

Candytuft, Carmine.............................. $35 \quad 350$

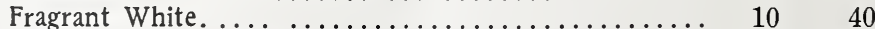

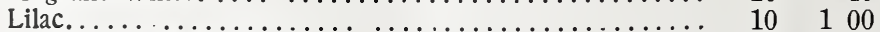

Rocket (Giant Empress) ..................... 20200

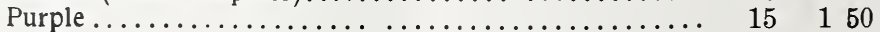

White................................... 10 40

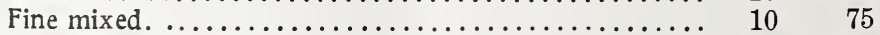

Canna, finest large-flowering, mixed.................... $15 \quad 150$

Canterbury Bell (Campanula medium) Single mixed............ $15 \quad 150$

Double Blue.............. $100 \quad 1000$

" Rose............. $100 \quad 1000$

" White................. $100 \quad 1000$

Double mixed........... $40 \quad 400$

Calycanthema Mixed...... $100 \quad 1000$ 


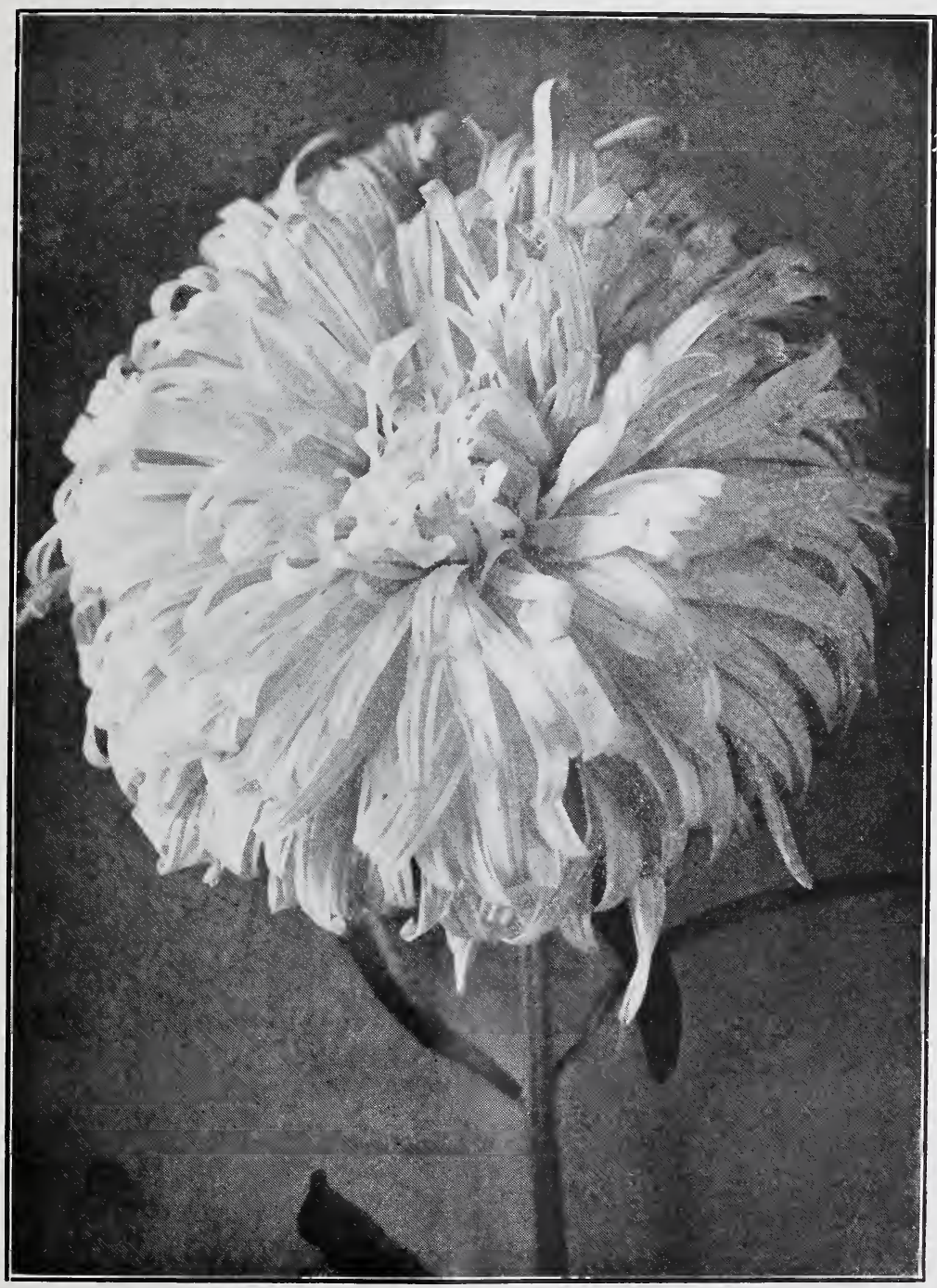

Giant Creco or Comet (See page 34) 


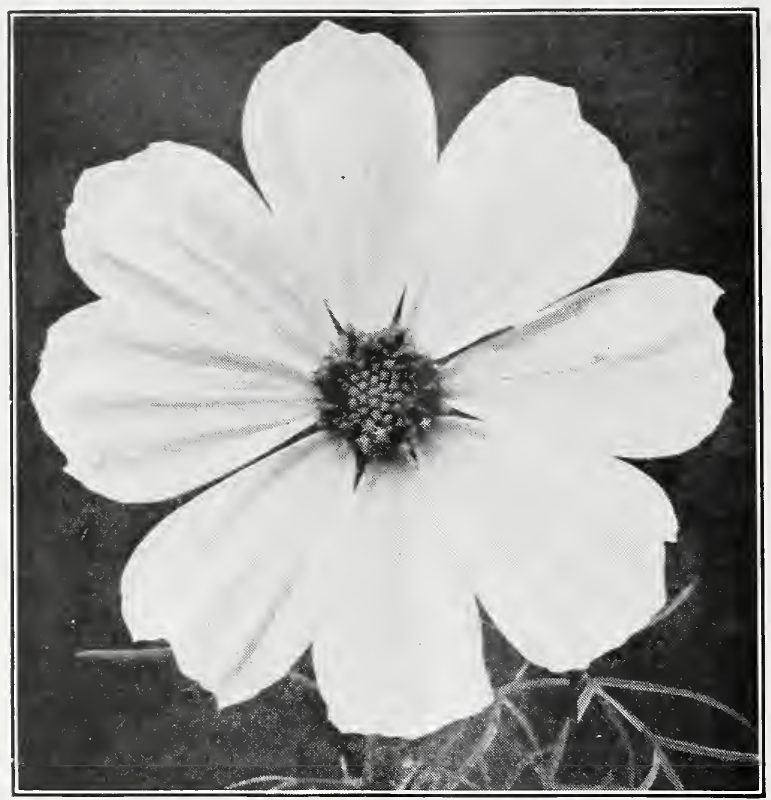

Cosmos

Per Oz. Per Lb.

Carnation, Double Picotee fine mixed.................. $\$ 150 \$ 1500$

“ $\quad$ Double dwarf Vienna, mixed, early flowering........... $100 \quad 1000$

“ Marguerite, finest mixed ...................... $75 \quad 750$

Castor Bean, Sanguineus ............................. $10 \quad 30$

" "

“ “ Mixed...................................... $10 \quad 50$

Celosia cristata (Cockscomb) dwarf mixed................. 75

Centaurea, Marguerite, white......................... 50

" Imperialis mixed .............................. $30 \quad 300$

“ Cyanus (Bachelor's Button) mixed ..................... $10 \quad 85$

“ " Double mixed (Bachelor's Button).............. $15 \quad 150$

Cineraria, Maritima candidissima.................... 20

Cobaea Scandens, violet-blue ............................ $30 \quad 300$

Columbine (Aquilegia) Californica hybrida ................. 150

“"

Convolvulus minor, mixed (Dwarf Morning Glory).............. 10

Cosmos, early flowering, Dawn, white....................... $20 \quad 175$

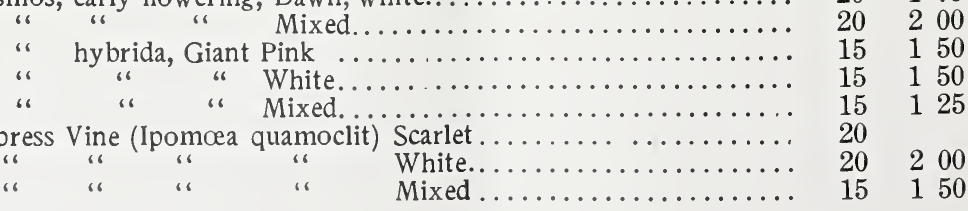




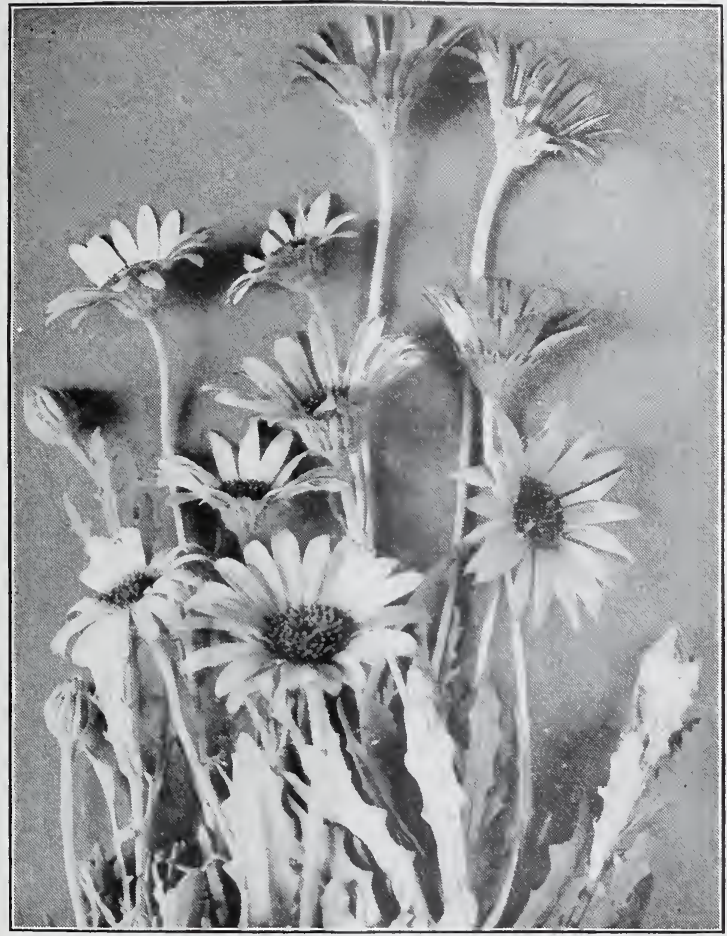

D.MORPHOTECA

Per Oz. Per Lb.

Dahlia, finest single mixed......................\$0 30

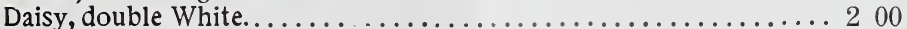

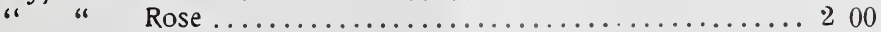

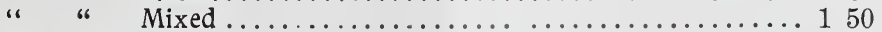

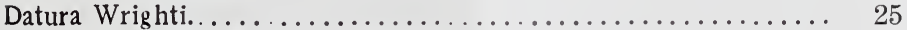

" chlorantha fl. pl., double yellow.................. $25 \$ 250$

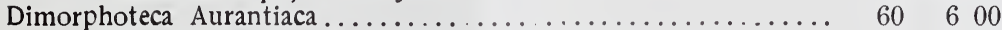

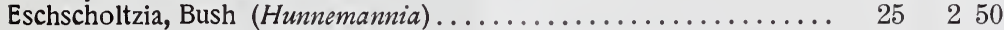

“ Californica, Deep Yellow (California Poppy) ........ $10 \quad 100$

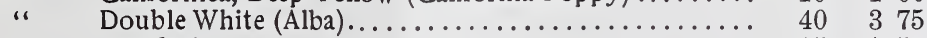

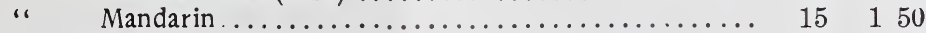

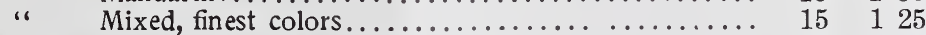

Forget-Me-Not, blue (Myosotis Alpestris)................. $30 \quad 300$

Four O'Clock (Mirabilis, Marvel of Peru) Mixed............... 10

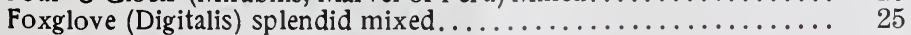

Globe Amaranth (Gomphrena globosa) Purple............... 15

" " " " " White................ 15

" "

" " " " 


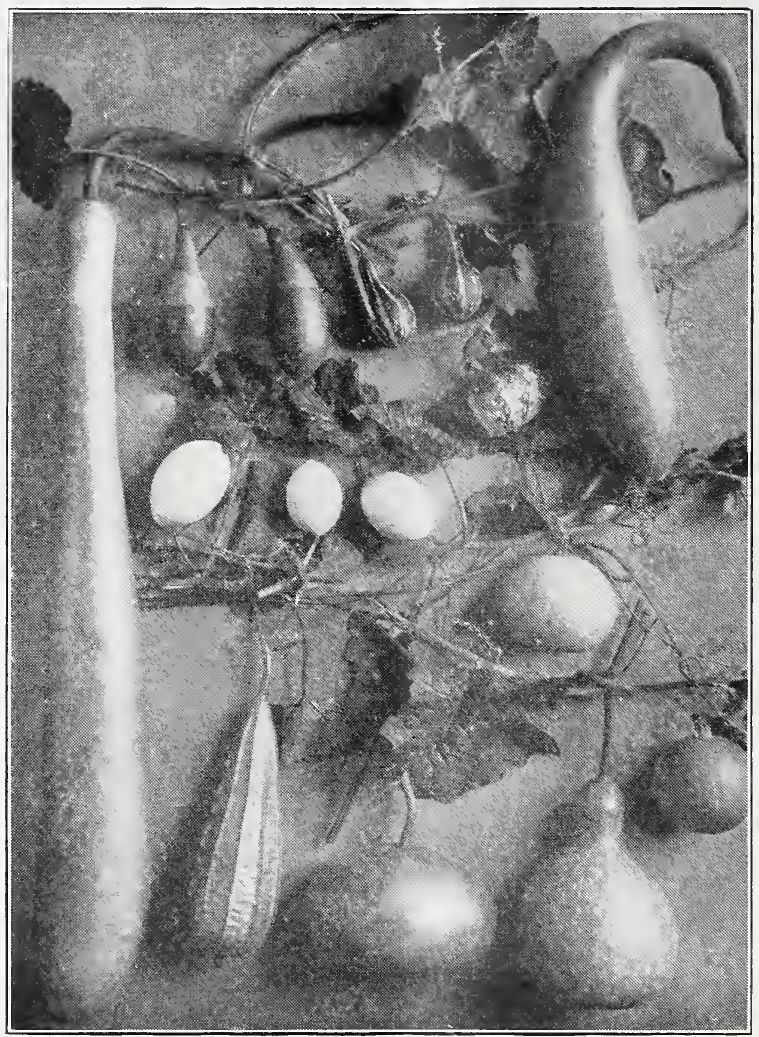

GOURDS

Per Oz. Per Lb.

Gourds, Apple shaped, early....................\$0 15

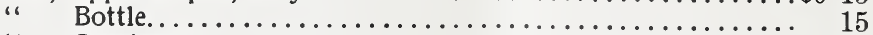

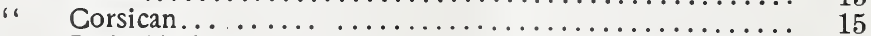

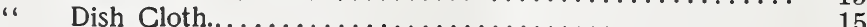

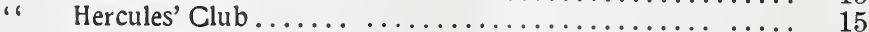

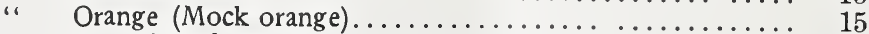

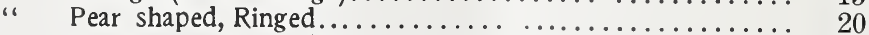

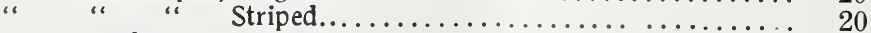

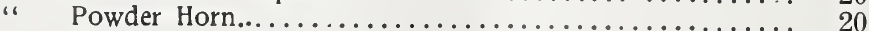

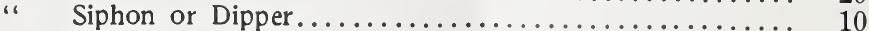

“ Mixed.................................. 10

Gypsophila, Elegans Large Flowering Pure White.............. 10

" " Mixed ........................ 10

Helichrysum monstrosum, largest double mixed $\ldots . . . . . . . . .$.

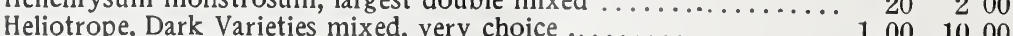

Hibiscus Africanus ................................. 1000 


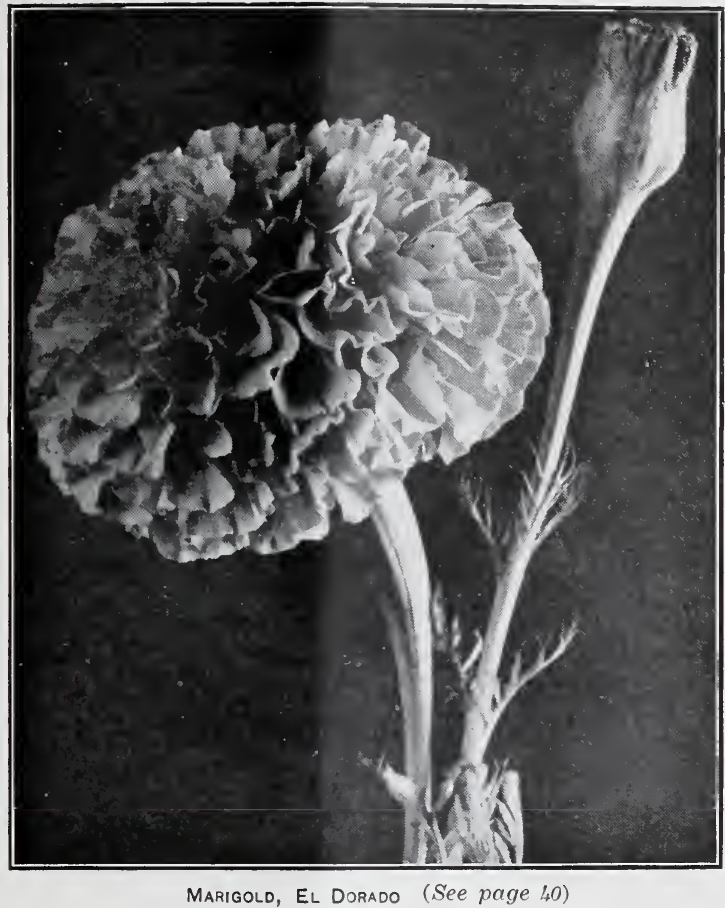

Hollyhock, Early Flowering Mixed.

Per Oz. Per Lb ......................\$1 00

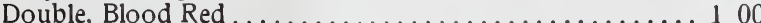

“ Deep Rose........ ...................... 100

“ Lemon Yellow.............................. 100

" $\quad$ Pink............................... 1 00

" Choice Mixed.

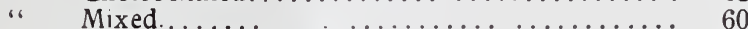

" Chater's Finest Mixed................... 150

Hyacinth Bean (Dolichos lablab) Alba, white................ $10 \quad 100$

" " " "

" " " " " Mixed .................... $10 \quad 100$

Ice Plant (Mesembryanthemum crystallinum)............... $10 \quad 100$

Ipomœa, Bona Nox (Evening Glory) .................... $10 \quad 100$

" Coccinea (Star Ipomøa)........................ $10 \quad 75$

" Limbata, mixed ............................. $10 \quad 75$

" Setosa (Brazilian Morning Glory).... ................ $20 \quad 200$

" Fine Mixed ...................................... $10{ }_{10} 100$

Job's Tears (Coix lachryma) ........................... $10 \quad 40$

Kochia Trichophylla . .

Lantana, finest French hybrids mixed..................... 20

Larkspur (Delphinium) double dwarf rocket, mixed............ $20 \quad 175$

Linum grandiflorum, rubrum (Scarlet Flax)................ $10 \quad 75$

Lobelia Erinus, Crystal Palcae. ........................ $100 \quad 1000$

" " Emperor William..................... 100 
Per Oz. Per Lb.

Love-in-a-mist (Nigella Damascena) Double Blue............\$0 $10 \$ \$ 50$

Lupin Cruikshanki ........................... $10 \quad 50$

Marigold, Meteor (Calendula officinalis, fl. pl.)............. 10 75

" Prince of Orange (Calendula officinalis, fl. pl.) ........ $10 \quad 80$

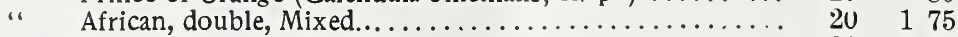

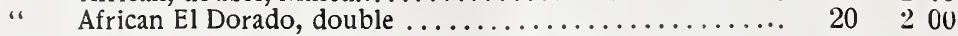

“ French, Legion of Honor (Little Bruwnie)........... 20

" Tagetes signata pumila, bright orange yellow......... $25 \quad 250$

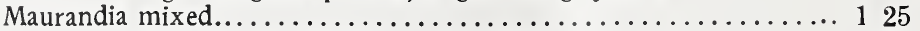

Mignonette, Sweet (Reseda odorata grandiflora).............. $10 \quad 60$

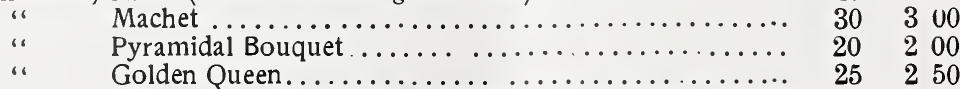

، Ruby, surpasses all other strains of the Machet class,

in large size of flower spike and splendid depth of color... $\quad 30 \quad 300$

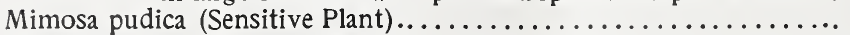

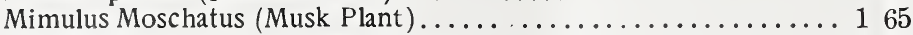

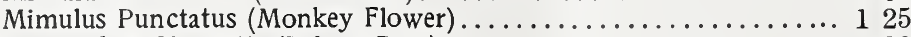

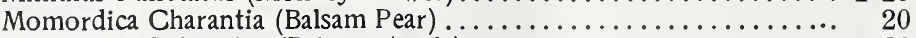

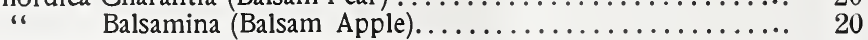

Moon Flower (Ipomoea Grandiflora Alba)................ 35

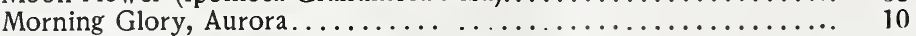

(Convolvulus major) Crimson ................ 10

Blue........................ 10

Purple............... 10

Striped ................ 10

White................ 10

Mixed ............. 10

Giant Japanese, Extra Select mixed ............. 15

250

1250

350

50

70

70

70

70

70

45

125

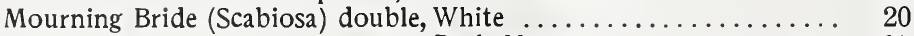

Dark Maroon................ 15

175

Mixed................ 15

175

\section{NASTURTIUMS}

\section{Dwarf Varieties}

(Tropoolum minor)

Per Oz. Per Lb.

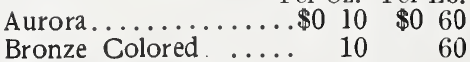

Crimson........... $10 \quad 60$

Chameleon.......... $10 \quad 125$

Empress of India..... $10 \quad 60$

King Theodore....... $10 \quad 60$

Pearl ............. $10 \quad 60$

Rose............. $10 \quad 60$

Scarlet........... $10 \quad 60$

Schilling's Striped ..... $10 \quad 60$

Yellow........... $10 \quad 60$

Mixed. (Tel. Cipher Itawa) sold out
Trailing Varieties

(Tropcolum Lobbianum)

Per Oz. Per Lb. Bright Yellow.......\$0 $10 \$ 060$ Brilliant .............. $10 \quad 85$

Chestnut Brown............ $10 \quad 85$

Giant of Battles......... $10 \quad 60$

Hemisphaericum...... $10 \quad 50$

Jupiter............... $10 \quad 50$

King of the Blacks..... $10 \quad 60$

Light Yellow, with maroon stain ......... $10 \quad 80$

Queen Wilhelmina..... $15 \quad 125$

Rose.............. $10 \quad 50$

Spitfire............... $10 \quad 125$

Mixed. (Tel. Cipher Izard) $10 \quad 50$

Nasturtiums, Madame Gunter's Hybrids.................. $10 \quad 40$

Trailing Chameleon..................... $10 \quad 135$

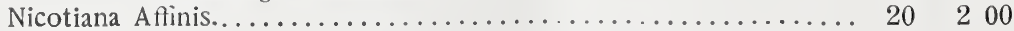




\section{PANSIES}

The variety of bloom, brilliancy of coloring and long keeping characteristics of pansies render them general favorites. We have been interested in the bringing forward of advanced types of very large varieties and our stocks are the very best obtainable.

Per Oz. Per Lb.

Pansy, large flowering, King of the Blacks.............. $\$ 0$

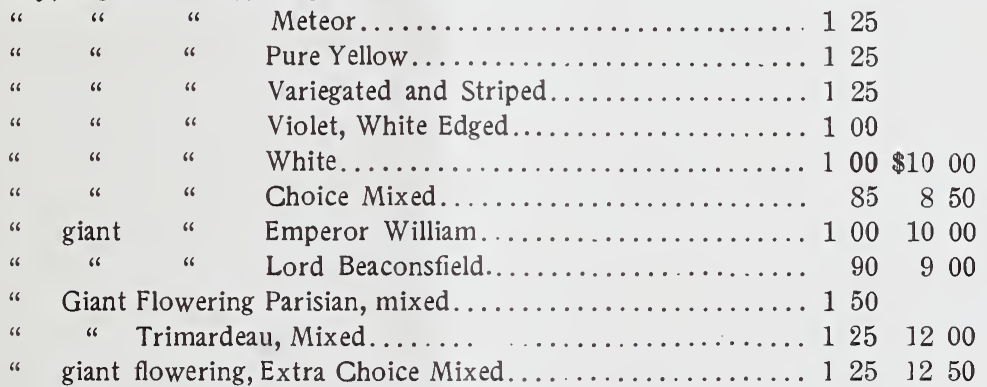

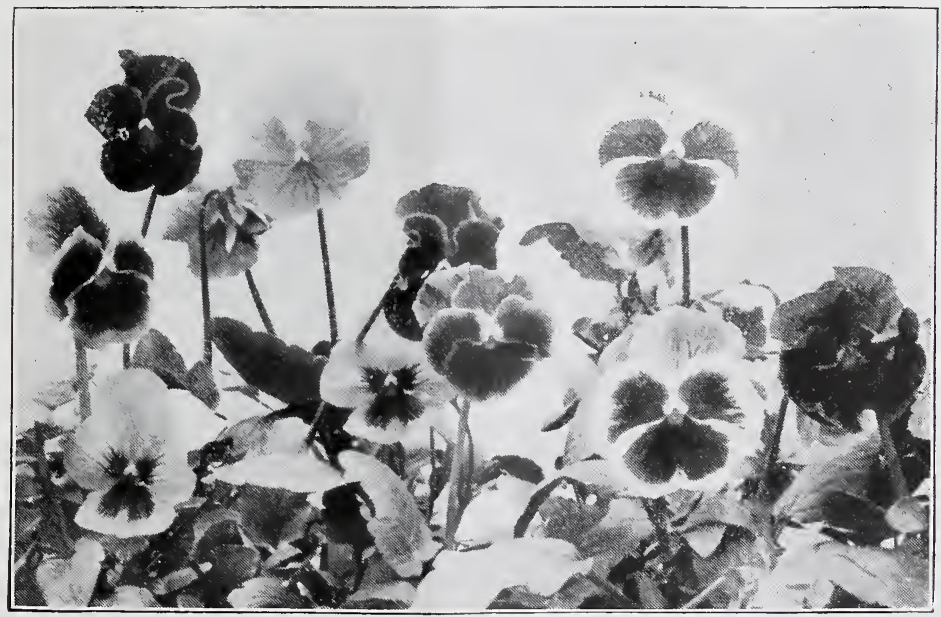

Giant flowered parisian pansies

Per Oz. Per Lb.

Peas, Everlasting (Lathyrus latifolius) Mixed...............\$0 $20 \quad \$ 1 \quad 75$

Petunia hybrida, Finest Striped and Blotched............... $50 \quad 500$

" " " Fine Mixed....................... $40 \quad 400$

" " $"$ Large flowered, Mixed ........... 11 oz. $\$ 0.60$.

“ “ $\quad$ Double, Mixed ................ $\frac{1}{18}$ oz. 2.50 
Per Oz, Per Lb.

Phlox Drummondi, large flowering, Alba, pure white.........\$0 60

\begin{tabular}{|c|c|c|c|c|}
\hline ، & “ & “" & “ & Black Warrior, deep maroon....... \\
\hline ، & “ & “6 & “6 & Isabellina, creamy yellow ......... \\
\hline ، & “ & “ & “ & Kermesina alba oculata........... \\
\hline ، & “ & “ & " & Lilac ................... \\
\hline ، & “ & “ & “ & Rosea, rose with distinct eye...... \\
\hline “ & “6 & “ & “ & Rosy Chamois................ \\
\hline “ & ، & “6 & “ & Scarlet, maroon center........... \\
\hline ، & “ & “6 & “ & Splendens, crimson, pure white eye. \\
\hline ، & " & “ & “ & $\ldots \ldots \ldots \ldots \ldots$ \\
\hline “ & “ & “6 & $\because$ & oice $n$ \\
\hline
\end{tabular}

Pink, China, double mixed (Dianthus Chinensis)............. 20

" Heddewig's Double Diadem Mixed................... 40

" "

“ Double Japan Mixed (Dianthus laciniatus fl. pl.).......... 50

Poppy, Shirley, extra fine mixed.................... $\quad 15$

" Tulip Flowered (Papaver glaucum) ................ 25

" Cardinal, double............................ 10

" Double White Fringed, extra fine................. 15

" Carnation, Double White........................ 10

" " Double Mixed........................ 10

" Double Choice Mixed........................... 10

" Orientale............................... 40

Portulaca, Single, large flowered, Alba, pure white........... 30

" " " " "

" " " " "

" Double, large flowered, mixed................. 225

Primrose, Evening (CEnothera) Lamarckiana............... 10

Primula, Sinensis fimbriata, choicest mixed......... $\frac{1}{8}$ oz. $\$ 2.00 . .1200$

Pyrethrum Parthenifolium aureum (Golden Feather).......... 25

Rhodanthe, Mixed............................. $\quad 30$

Salpiglossis, fine mixed hybrid varieties............... 40

Salvia splendens (Flowering Sage) .................... 175

Smilax (Myrsiphyllum asparagoides) .................. 20

Snapdragon (Antirrhinum) large flowering, fine mixed......... 25

Stock, Early Flowering Brompton Mixed................ 250

" Double German Ten Weeks, Mixed................. 50

" " " " " "

" ، " "

" Brompton, mixed.......................... 350

Sunflower, Globosus fistulosus, double................ 10

300

400

2500

200

250

500

1200

" Double Chrysanthemum flowered.............. 15

Stella ................................ 15

Sweet Peas, see pages $44,45,46,47$. 
Per Oz. Per Lb.

Sweet William (Dianthus barbatus) Pure White............\$0 15

" " " " Velvety Maroon............. 15

" " " " "

" " " "

Verbena hybrida, Auricula flowered.................... 75

$\$ 090$

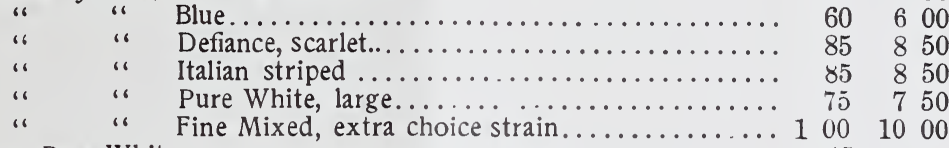

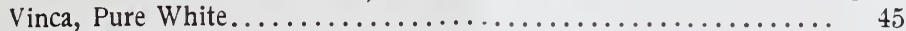

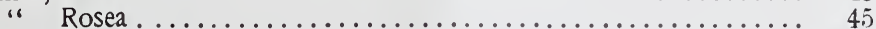

" Rosea Alba............................. 45

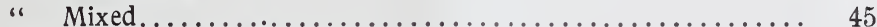

Wallflower, Harbinger, Early Brown ................... 15

" Mixed Double, extra fine. .................. 175

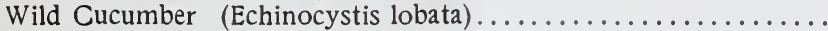

Zinnia, Elegans Double, Black Purple, Magenta, Orange, Scarlet, or Yellow, in separate colors, each........ 30

White ............................... 25

Striped or Zebra, mixed ............ 30

" Choice Mixed.................. 25

Large Flowered Double Mixed.............. 40

" Double, Pompon, finest mixed..................... 30

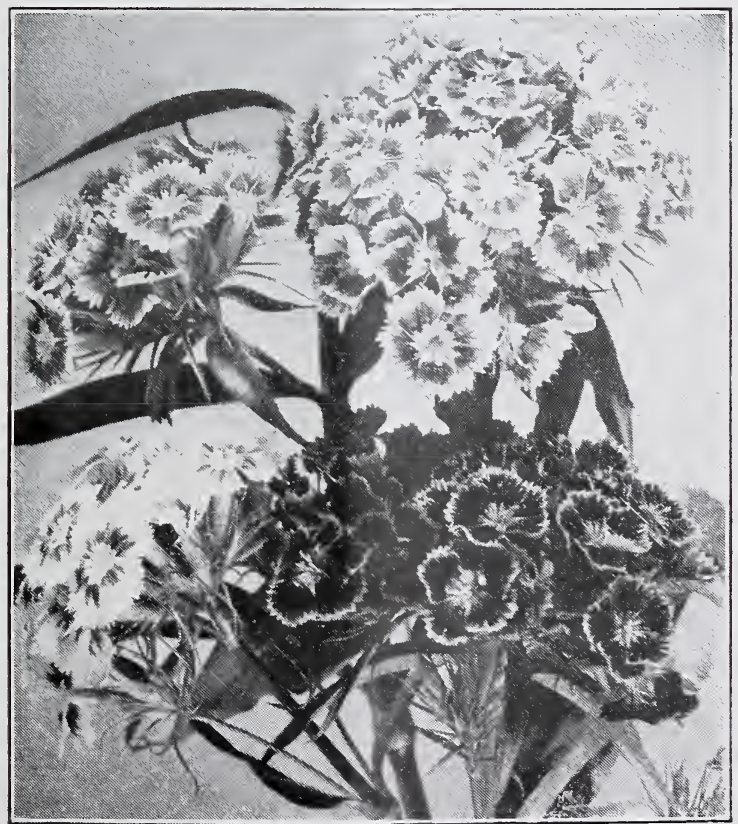

SWEeT WiLLiam 


\section{SWEET PEAS}

Our stocks are absolutely unsurpassed in selection, purity and vitality.

Sweet Peas 10c. per oz. unless otherwise stated.

Not less than one-fourth pound at pound rate.

\section{EARLY FLOWERING SPENCER}

Yarrawa, bright rose pink. A most desirable variety

Tel. Cipher Per Oz. Per Lb.

for winter flowering indoors............Wauna....\$1 00

\section{SPENCER VARIETIES}

Apple Blossom Spencer, bright rose with primrose

wings tinted rose-carmine; very large............. Yadkin ..... 15

Asta Ohn, suffused lavender or tinted mauve, some-

times with pinkish tinge, large and wavy $\ldots . .$. Yamhill.... $20 \quad 175$

Aurora Spencer, orange-rose striped on white...Yankton.... $15 \quad 150$

Blanche Ferry Spencer, rose-pink standard, white

wings, tinted light pink; very large and wavy....Tulare..... $20 \quad 200$

Captain of the Blues Spencer, purple with blue wings, lighter than Captain of the Blues. ......Waseca..... 20

Countess Spencer, bright pink, Spencer type, very large, long stems. Especially desirable.........Vance..... $15 \quad 150$

Dainty Spencer, white suffused and edged with rosepink, very free blooming. ..................Washita... $20 \quad 200$

Dobbie's Cream, a very superior cream or primrose. Wasatch ... $20 \quad 200$

Elfrida Pearson, one of the best light blush pinks..Waskom... $25 \quad 225$

George Herbert, large rose-crimson or rose-carmine Spencer, open form and wavy ...............Washoe... $15 \quad 15$ J

Helen Lewis, large crimson-orange with wings of orange-rose or orange-salmon pink, Spencer type..Wallace... $20 \quad 175$

Illuminator, bright glowing salmon..........W Ward..... $30 \quad 300$

King Edward Spencer, brilliant crimson-scarlet, one of the best bright red Spencers..............Watauga... $20 \quad 175$

King Manoel, deep purplish maroon .......... Watab..... $20 \quad 225$

King White, one of the best yet introduced .....Weld..... $25 \quad 250$

Loyalty Spencer, bright blue flaked and striped on white ........................................ $20 \quad 200$

Margaret Atlee, a very desirable deep cream pink..Wekiwa .... $\quad 25 \quad 250$

Margaret Madison (New), soft light azure blue or pale lavender..........................Welaka... $30 \quad 325$

Mrs. Hugh Dickson, light salmon pink..........Welga..... $20 \quad 200$

Othello Spencer, very large, beautiful deep maroon..Yell. .... $\quad 20 \quad 225$

Primrose Spencer, clear, distinct large, wavy; one of the best primrose or cream and ivory varieties... Waukesha.. $20 \quad 200$

Royal Purple, rich royal purple .............Waukon ... $35 \quad 350$

Scarlet Emperor, brilliant deep scarlet......... Wehrum ... 25 250

Stirling Stent, rich metallic salmon ............Wecota.... $20 \quad 225$

Thomas Stevenson, brilliant orange-scarlet Spencer.Yetter..... $20 \quad 200$

White Spencer, pure white, an especially attractive Spencer of very large size. ............... Yolo..... 


\section{SWEET PEAS-Continued}

\section{GRANDIFLORA VARIETIES \\ ROSE STANDARD WITH WHITE WINGS (BICOLOR)}

Extra Early Blanche Ferry, standard bright rose-pink, with

Tel. Cipher Per Lb nearly white wings. We are headquarters for this, the earliest

Sweet Pea for forcing or growing outdoors.

Blanche Ferry, attractive rose-pink; wings white tinged pink.

The typical pink and white sweet pea............... Tucker....

\section{WHITE}

Dorothy Eckford, pure white, beautifully shell shaped. .....Tuolumne.

Earliest White, the earliest white sweet pea; very desirable for forcing. ........................... Tuscola... 100

Emily Henderson, clear white, well formed ........... Tyler..... 50

Mont Blanc, good form and clear white color..........Umatilla.. 100

\section{PRIMROSE OR VERY LIGHT YELLOW}

Hon. Mrs. E. Kenyon, a clear shade of primrose......... Utah.....

Mrs. Collier, large, light primrose; lighter than Hon. Mrs. E.

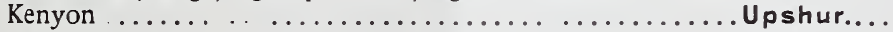

LIGHT PINK

Dainty, white, shaded lightly and edged with light pink.....Valverde...

Katherine Tracy, a soft but brilliant pink............Vernon.... 60

Lovely, deep pink, shading lighter at edges............ Vinton.... 50

Mrs. F. J. Dolansky, pure light pink desirable for early flowering indoors.................................... 100

Prima Donna, a brilliant yet soft shade of pink.........Wabash ...

\section{ORANGE-PINK}

Bolton's Pink, orange-pink veined rose, semi-hooded.......Waller... 60

Miss Willmott, orange-pink; wings rose, tinted orange......Walton.... 60

St. George, the brightest orange-pink, wonderfully brilliant... Walworth . 90

SHADES OF RED

King Edward VII, bright crimson-scarlet. ...........Wayne.... 60

Prince of Wales, a pure rose-crimson of large size.........Wells .... 60

Queen Alexandra, large, bright red, almost scarlet........Wetzel.... 60

PINK AND RED STRIPES

America, carmine striped and splashed on white ground.....Wapello... 50

Aurora, large, delicate orange-salmon-pink over white.......Warren... 50 
D. M. FERRY \& CO., DETROIT, MICH.

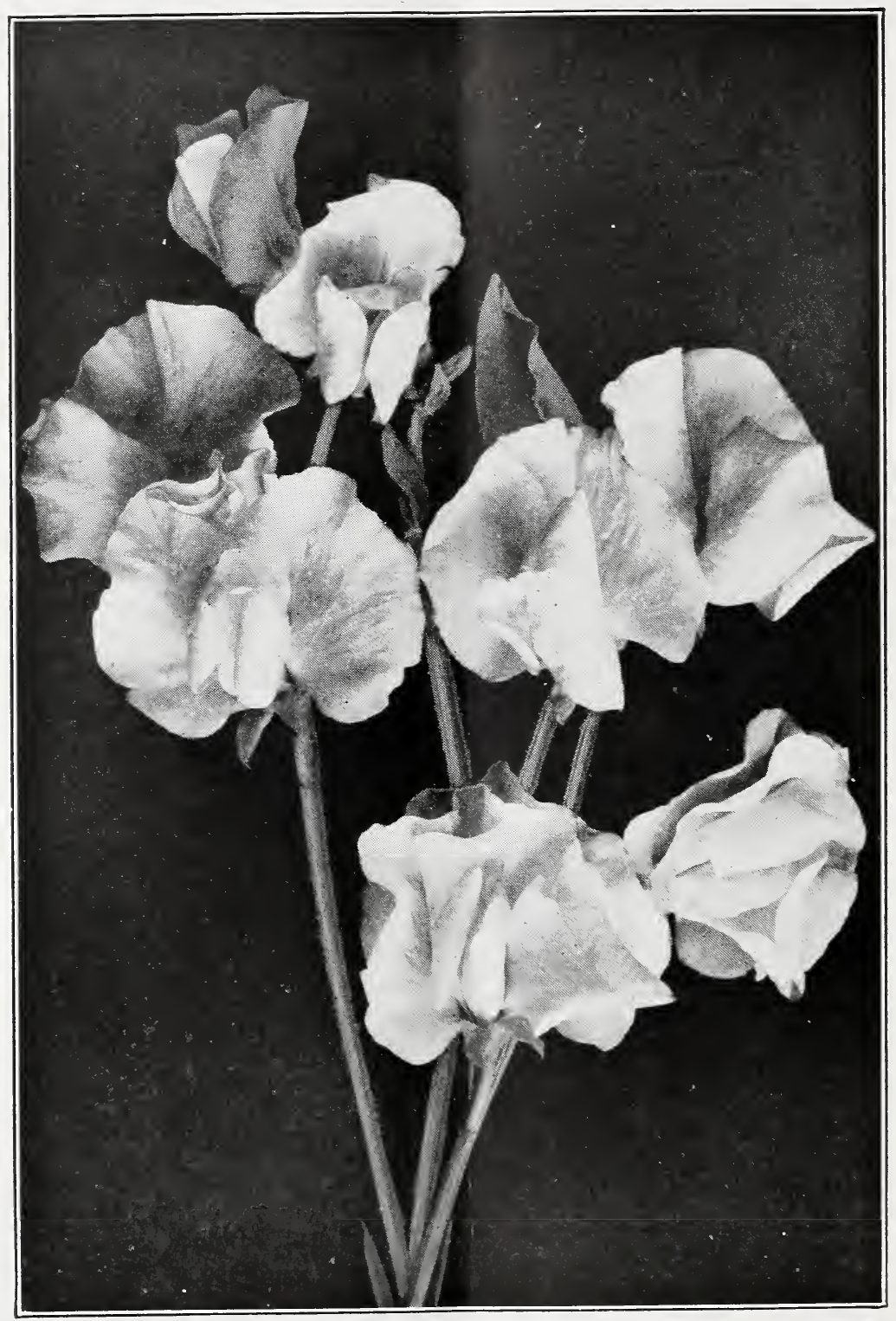




\title{
SWEET PEAS-Continued
}

\author{
GRANDIFLORA VARIETIES
}

LAVENDER AND LIGHT BLUE

Tel. Cipher Per Lb.

Countess of Radnor, a beautiful shade of lavender........Whitley..... \$0 50

Helen Pierce, veined and mottled bright blue on ivory white. Wilkes..... 60

Lady Grisel Hamilton, similar to Countess of Radnor, but

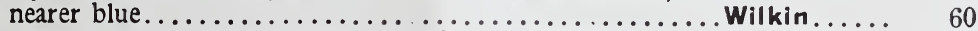

Lottie Eckford, white shaded lilac, edged magenta-blue..... Windham .. 50

Mrs. Chas. H. Totty, large early flowering lavender.......Winn...... 100

Mrs. George Higginson, Jr., clear, delicate lilac blue........ Winona.... 60

MAUVE AND PURPLE

Mrs. Walter Wright, a large hooded rosy purple ......Wise...... 50

BLUE AND PURPLE

Duke of Westminster, purple shading to violet.......... Woodbury..

Flora Norton, hooded form, clear light blue ............Woodson...

Lord Nelson, rich blue, deeper than Navy Blue..........Woodward.

\section{BLUE AND MAROON STRIPES}

Senator, purplish maroon and indigo striped on light ground. Wythe.....

\section{VIOLET AND MAROON}

Black Knight, glossy deep maroon, one of the darkest...... Yakima .... 60

Othello, very dark maroon, practically self colored....... Yoakum....

CHOICE MIXED. This mixture has been most car efully grown from our selected stock seed and it will afford a splendid variety of blooms of improved forms and in the widest possible range of desirable colors.

Tel. Cipher Zapata. Per Lb. 50c; Per 100 Lbs. $\$ 45.00$

FERRY'S SUPERB MIXED. We make up this mixture by combining in carefully considered proportions twenty-one separate named varieties, each the very choicest of its class. Nowhere else can one procure a more magnificent mixture of finely formed and beautifully colored, large flowering Sweet Peas.

Tel. Cipher Yuma. Per Lb. 65c; Per 100 Lbs. $\$ 60.00$

SPENCERS MIXED. In this splendid mixture will be found a wide range of colors of this comparatively new class. The flowers are of remarkably large size and wonderfully attractive form.

Tel. Cipher Young. Per Oz. 15c; Per Lb. \$1.50

FERRY'S SUPERB SPENCERS MIXED. We make up this magnificent mixture by combining eighteen separate named Spencer varieties, each of which our trials have repeatedly shown to be the very best of its class. Unsurpassed as a mixture of this superb class and showing the most desirable range of colors and the finest forms. No better mixture in all the world than this. 


\section{GARDEN TOOLS}

Send for Illustrated and Descriptive Catalogues of Garden Tools for 1917

Packed or boxed ready for shipment, and delivered at express office or depot at Detroit, Mich.

Tel. Cipher Price

Ames' New Universal Garden Drill, No. $17 \ldots \ldots \ldots \ldots \ldots \ldots \ldots \ldots \ldots$ Taceo........... \$ 575

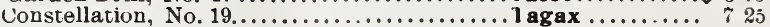

Planet Jr., No. 3, Hill and Drill Seeder.......................Teho......... 950

"4, Combined Hill and Drill Seeder, Wheel Hoe,

Cultivator and Plow...., ............................Temere......... 1000

“4, Hill and Drill Seeder. Plain (as a drill only) ........Tempsa......... 800

“ 25, Combined Hill and Drill seeder and Double Wheel

Hoe, Cultivator and Plow..................... Teneo.......... 1200

" 31 , Combined Drill Seeder and Wheel Hoe............... Tenor............ 650

" 11 , Double Wheel Hoe................................. Tergo............ 8 . 25

" $12, \quad$ " " $"$ "

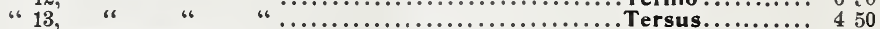

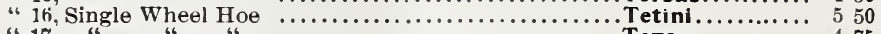

" 17, " " "

" 18, “ " " "

" "19, " " "

" 38, " " " Dise Hoe, Cultivator and Plow....... Theto............ 650

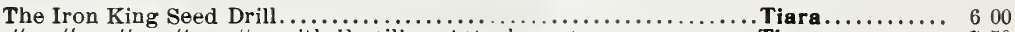

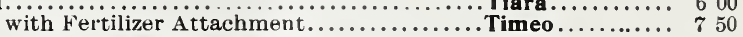

Excelsior Weeding Hook.........................15 cts. each.. Titubo..per doz. 125

Hazeltine Hand Weeder and Seraper......................20 " ".Toga...." ". 2 " 25

Christy Garden Weeder. .................................. " "

Pressed Steel Garden Trowel ........................ " " .Tonum.. " " 125

\section{SEED BAGS}

We annually manufacture for our own use millions of Seed Bags and are in a position to offer to the trade first quality ready made bags at prices as reasonable as the same grade of goods can be purchased anywhere.

Samples of all sizes furnished on application.

\section{Printing Extra, at Cost}

No. 1 Miniature Pockets, Canary .......... $7 / 8 \times 2 \mathrm{l} / 4$ inches... Per $\$ 060$

" 3 White Coated Paper.............. 21/8 x $31 / 4 \quad$ " .... 75

" 7 White Coated Paper.............. $31 / 8 \times 15$ x $4 \frac{1 / 2}{2}$ " ... 115

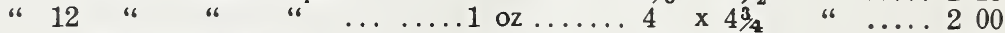

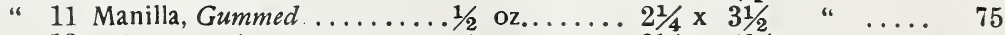

" 13 " "

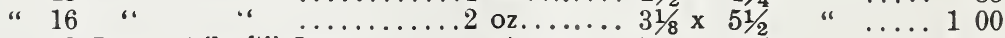

" 33 Brown "Kraft" Paper ....... pint...... 5 x $7 \frac{1}{2}$ " $\ldots . .250$

" 36 "

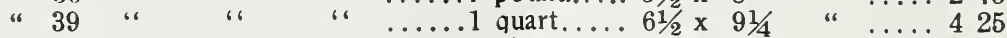

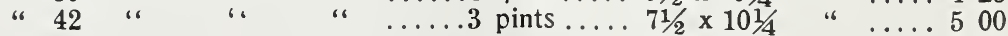

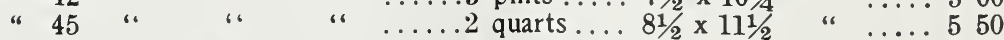

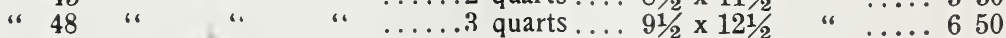

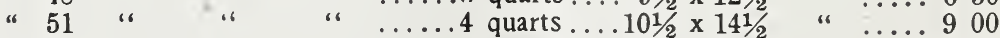

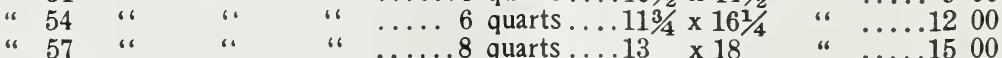

Bags Nos. , to 16 have flaps; only Nos. 11,13 and 16 are gummed. 


\section{TELEGRAPHIC CIPHER}

By making use of the following Telegraphic words for weights, together with the Telegraphic Cipher attached to each article in this list, our customers will be able to order by wire at trifling expense. The Telegraphic words for QUANTITIES must always PRECEDE the cipher words for Seeds, but if the same quantity of several articles is needed, the word for that quantity should be placed before the word for the first article only. For example:

"Freight Adjust Lagrange, Lemhi, Lorain." Means "Ship by first freight One Hundred Pounds each of Ferry's First and Best, Telephone and Champion of England Peas."

"Express Above Caddo, Cannon, Catoosa." Means "Send by first express Two Pounds each of Early Jersey Wakefield, Early Winnigstadt and D. M. Ferry \& Co's Premium Late Flat Dutch Cabbage."

TELEGRAPHIC WORDS FOR WEIGHTS

Quantity OUNCES

$1 / 8 \ldots$. Aback

$1 / 4$.....Abaft

$1 / 2 \ldots$. Abase

$3 / 4$.... Abash

1..... Abate

2......Abbey

3......Abbot

POUNDS

$1 / 4$.....Abhor

$1 / 2$.....Abide

$3 / 4 . .$. Abolish

1......Abound

2......Above

3..... Abrade

4......Abrupt

5......Abscind

6.... Abscond

7 ......Absent

8.....Absolve

9 ......Absorb

10 ......Abstain

11 ....Abstract

12 .....Absurd

$13 . .$. Abuse

14 ......Accede

15 ......Accent

16 ......Accept

17 .....Access

18 ....Accost

19 ......Account

20 ......Accredit

21 .....Accuracy

22 ......Accuse

23 .......Acquaint

24.... Acquire

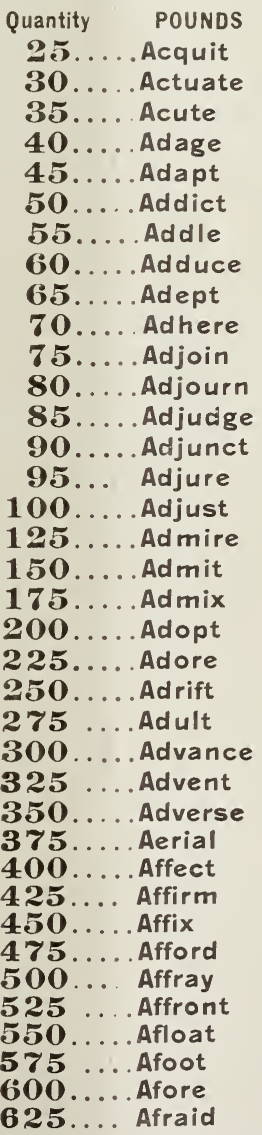

Quantity POUNDS

650 . Against

675..Aggress

700 . Aghast

725. Agnate

750..Alarm

775 . Albeit

800. Alcove

825. Alert

850 . Alight

875. Allay

900 . Allege

925 . Alloy

950 . Allude

975. Allure

1000 . Alpha

1500. Alpine

2000. Alter

2500 .. Alvine

3000 . . Amass

3500. Amaze

4000. Ambush

4500 . Amend

5000 . Amenity

5500 ..Amiss

6000. Ample

6500 . Amuse

7000 . Analyze

7500 . Anchor

$\mathbf{8 0 0 0 . . A n c i e n t}$

8500 . Adroid

9000 .. Angler

9500 . Anile

10000 . Anthem

15000 . Antic

20000..Antler

25000 . Antelope

NOTE.-The above Code for TELEGRAMS only-Not for orders by mail. 


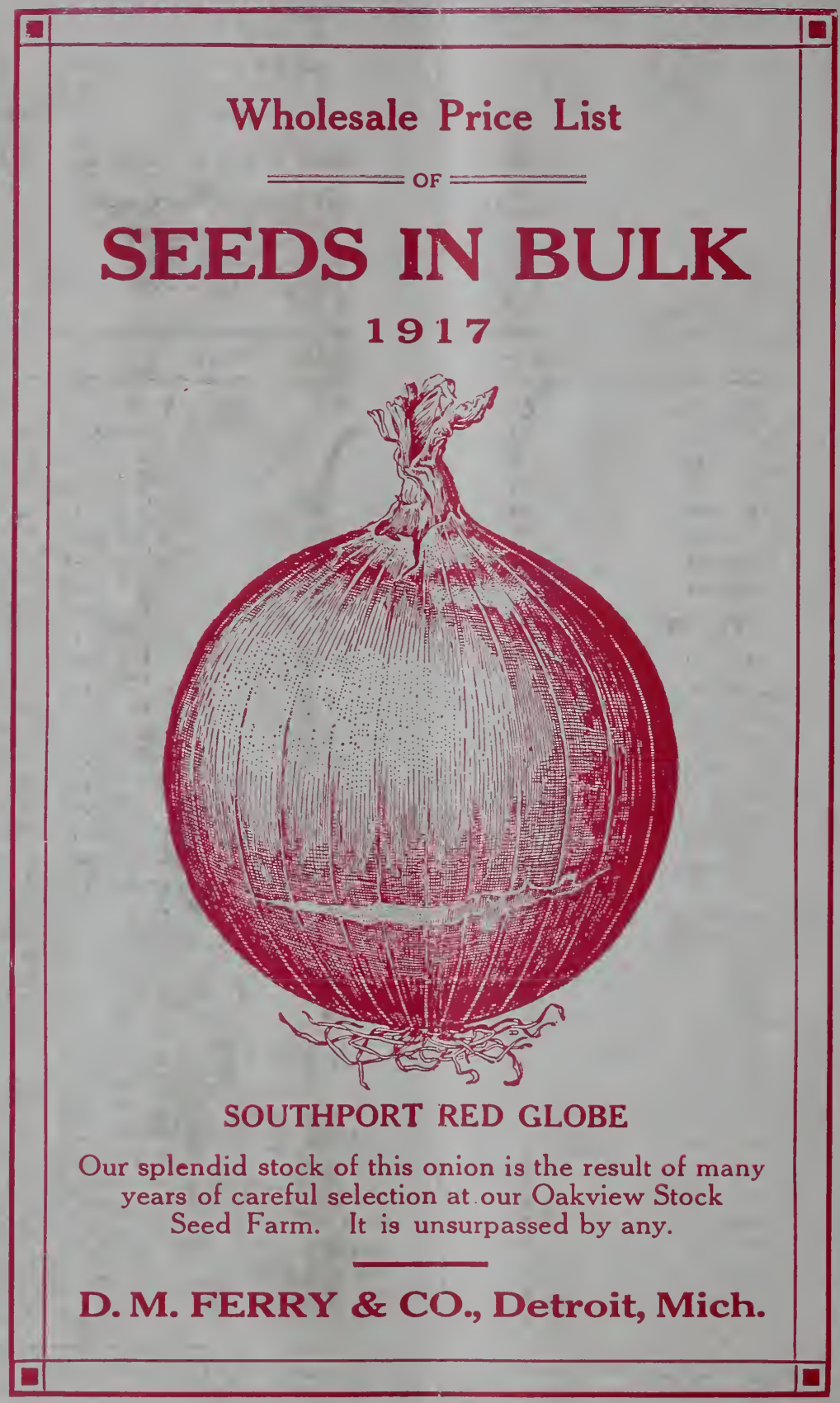

